\title{
Medidas difusas e integrales difusas
}

\author{
Gilberto Arenas-Díaz and Édgar René Ramírez-Lamus
}

Received: 05-11-2012 Accepted: 28-01-2013 Published on line: 22-02-2013 Edited by Alberto Acosta

Fuzzy measures and fuzzy integrals. Abstract. This is a study of fuzzy measures and fuzzy integrals; it presents some of the phenomena where they are used. It exposes how the concept of fuzzy measure is introduced and from it, the notion of fuzzy integral is presented. Properties of fuzzy measures are established and they are classified according to additive property and $\lambda$-measures, while the probability, plausibility, credibility, possibility and necessity measures are observed as classic examples of the classification completed. The two main fuzzy integrals were analyzed: the Sugeno integral and the Choquet integral, given the application of these integrals is made on finite sets, a comparison between them for the finite case is performed using the concept of equiordered functions. We present two examples in which fuzzy measures and fuzzy integrals are used in the classification of individuals and in quality assessment. It also describes some phenomena where they are applied. Classic measures are used in certain special cases of uncertainty based on randomness. The use in certain contexts of fuzzy measures (non-additive) and fuzzy integrals offer a more flexible and realistic focus in modeling uncertainty.

MSC2010: Primary 28E10; Secondary 26E50.

Keywords: fuzzy measure, $\lambda$-measure, Choquet integral, Sugeno integral, equiordering function.

Escuela de Matemáticas, Universidad Industrial de Santander, A.A. 678, Bucaramanga, Colombia.
Resumen. Se presenta un estudio de las medidas difusas y de las integrales difusas, y se muestran algunos fenómenos donde estas son utilizadas. Se expone cómo es introducido el concepto de medida difusa y cómo a partir de él se introduce la noción de integral difusa. Se establecen propiedades de las medidas difusas y se clasifican de acuerdo a la propiedad aditiva y las $\lambda$-medidas, se observa cómo las medidas de probabilidad, plausibilidad, credibilidad, posibilidad y necesidad son ejemplos clásicos en la clasificación realizada. Se hace un análisis de las dos principales integrales difusas: integral de Sugeno e integral de Choquet, dado que la aplicación de estas integrales se hace sobre conjuntos finitos, se realiza utilizando el concepto de función equiordenada una comparación de ellas para el caso finito. Se presentan dos ejemplos donde se utilizan las medidas difusas e integrales difusas en los procesos de clasificación de individuos y evaluación de calidad. También se describen algunos fenómenos donde ellas son aplicadas. Las medidas clásicas son usadas en ciertos casos especiales de incertidumbre basados en la aleatoriedad. La utilización en determinados contextos de medidas difusas (no aditivas) y de las integrales difusas ofrece un enfoque más flexible y realista para modelar la incertidumbre.

Resumo. Apresentamos um estudo de medidas difusas e integrais difusas, e mostramos alguns fenómenos onde são utilizadas. Expõe-se como é introduzido o conceito de medida difusa e como a partir daí se introduz a noção de integral difusa. Estabelecem-se propriedades das medidas difusas e classificam-se de acordo com a propriedade aditiva e as $\lambda$-medidas, observa-se como as medidas de probabilidade, plausibilidade, credibilidade, possibilidade, e necessidade são exemplos clássicos da classificação feita. Faz-se uma análise das duas principais integrais difusas: integral de Sugeno e integral de Choquet, uma vez que a aplicação destas integrais é feita sobre conjuntos finitos, realiza-se utilizando o conceito de função equiordenada comparando-as com o caso finito. Apresentam-se dois exemplos que usam medidas difusas e integrais difusas nos processos de classificação dos indivíduos e de avaliação de qualidade. Também se descreve alguns fenómenos onde elas são aplicadas. As medidas clássicas são usadas em certos casos especiais de incerteza baseados em aleatoriedade. O uso em determinados contextos das medidas difusas (não aditiva) e integrais difusas fornece uma incerteza de modelagem mais flexível e realista. 


\section{Introducción}

Intentando dar otro enfoque en la generalización del concepto de medida, en los años setenta del siglo pasado Michio Sugeno define por primera vez los conceptos de "medida difusa e integral difusa" (Sugeno, 1974). Una de las características principales de las medidas difusas es que no requieren la propiedad de la aditividad, en contraste con las medidas clásicas; por eso también son llamadas medidas no aditivas. Las medidas difusas según Sugeno, se obtienen mediante la sustitución de la aditividad de las medidas clásicas por propiedades más débiles como la monotonía, la continuidad por abajo y la continuidad por arriba. El nombre acuñado por Sugeno fue dado porque él intentaba comparar las medidas de probabilidad con las funciones de pertenencia de los conjuntos difusos. Sugeno intentó generalizar las medidas clásicas a las medidas difusas, tratando de hacer un proceso análogo al realizado en la generalización de los conjuntos clásicos en conjuntos difusos. Debido a esta analogía fue dado el nombre de dichas medidas. Es de mencionar que la noción de conjunto difuso fue propuesta por Zadeh (1965) con el objetivo de definir conjuntos que no tienen fronteras bien definidas.

Aunque el término de medida difusa ha sido aceptado por la comunidad científica, también ha servido para confusiones, ya que la palabra "difusa" no necesariamente implica que la medida se aplique a conjuntos difusos o que la medida sea difusa en el sentido de dicha teoría. Concretamente, una medida difusa es una función monótona no negativa de valores definidos en "conjuntos clásicos". Actualmente, en los libros y artículos sobre este tema, el término de "medidas difusas" ha sido sustituido por el término de "medidas monótonas", "medidas no aditivas" o "medidas generalizadas" (Asahina et al., 2006; Denneberg, 1994; Song, 2005; Song y Li, 2005; Murofushi y Sugeno, 2000; Wang, 1992; Wang y Klir, 2009). Cuando las medidas difusas son definidas sobre conjuntos difusos, se habla de medidas monótonas fuzzificadas (Wang y Klir, 2009).

A pesar de las muchas aplicaciones de la teoría clásica, cada vez más, se reconoce que ella está limitada por el requisito de la aditividad de las medidas. Exigir la aditividad en la medición de una propie- dad de un conjunto de algún tipo es básicamente suponer que no hay interacción entre los elementos del conjunto y la medida de ellos. Sin embargo, hay problemas en los cuales sí hay interacción entre los elementos del conjunto y su medida. Sea $(X, \mathscr{A})$ un espacio medible (al par $(X ; \mathscr{A})$, donde $X$ es un conjunto y $\mathscr{A}$ es una $\sigma$-álgebra de $X$, es llamado espacio medible y a los elementos de $\mathscr{A}$ se les llama conjuntos medibles). Se puede encontrar un estudio detallado de estos conceptos en Ash (2000) y Folland (1999). Se dice que una función $\mu$ describe una interacción entre los elementos de los conjuntos $A, B \in \mathscr{A}$ con $A \cap B=\varnothing$, si sucede alguna de las siguientes situaciones:

1. Se expresa una interacción positiva (cooperación, mejora, ampliación) entre $A$ y $B$ en términos de la propiedad medida, si $\mu(A \cup B)>\mu(A)+\mu(B)$.

2. Se expresa una interacción negativa (incompatibilidad, rivalidad, inhibición) entre $A$ y $B$ en términos de la propiedad medida, si $\mu(A \cup B)<\mu(A)+\mu(B)$.

3. Se expresa que no hay interacción entre $A$ y $B$ en términos de la propiedad medida, si $\mu(A \cup B)=\mu(A)+\mu(B)$.

Obsérvese que las medidas clásicas solo pueden capturar la tercera situación, que es aplicable sólo a las propiedades que no son interactivas.

La utilización de medidas no aditivas en determinados contextos de aplicación, ofrece un enfoque más flexible y realista a una amplia gama de problemas. Por ejemplo, si se considera un conjunto de trabajadores $X$ en un taller, que son involucrados en la elaboración de productos de un tipo específico, y se supone que el conjunto $X$ está dividido en subgrupos $G_{1}, G_{2}, \ldots, G_{N}$, donde $\mu\left(G_{i}\right)$ indica el número de productos fabricados por cada grupo $G_{i}$ dentro de una determinada unidad de tiempo, entonces se puede presentar que cuando los grupos trabajan por separado, la función $\mu$ es una medida aditiva. Sin embargo, cuando algunos de los grupos trabajan juntos y hay cooperación eficiente entre ellos, la medida es superaditiva. Por el contrario, si la cooperación es ineficaz, la medida es subaditiva. 
Como se ha dicho, en general, las medidas difusas pierden la propiedad de aditividad, que aparentemente las hace mucho más flexibles que las medidas clásicas. Pero debido a esto, es díficil desarrollar una teoría general de las medidas difusas sin ninguna condición adicional.

En el trabajo pionero de Sugeno se introduce una medida difusa llamada $\lambda$-medida o medida de Sugeno, que permite generalizar la propiedad aditiva de las medidas de probabilidad (Banon, 1981; Geronimo, 1988):

$$
g_{\lambda}(A \cup B)=g_{\lambda}(A)+g_{\lambda}(B)+\lambda \cdot g_{\lambda}(A) \cdot g_{\lambda}(B),
$$

siempre que $A \cap B=\varnothing$, donde $\lambda \in(-1, \infty)$. Posteriormente, Shafer (1976) introduce dos medidas difusas llamadas medidas de credibilidad y plausibilidad, fundamentales en el desarrollo de la teoría de la evidencia; este trabajo fue motivado por la investigación previa de Dempster sobre probabilidades inferior y superior, investigación enmarcada también dentro de la teoría de la evidencia, y conocida como la teoría Dempster-Shafer (Shafer, 1976; Dempster, 1967a, 1967b).

Más adelante se introduce una nueva medida difusa, llamada medida de posibilidad, que satisface $\Pi(A \cup B)=\operatorname{máx}\{\Pi(A), \Pi(B)\}$ (Zadeh, 1978). Una medida de posibilidad es un caso particular de una medida de plausibilidad. También definen una medida difusa dual a la medida de posibilidad, llamada medida de necesidad, y que satisface la ecuación $\operatorname{Nec}(A)=1-\Pi\left(A^{c}\right)$, donde $A^{c}$ denota el complemento del conjunto $A$. Una medida de necesidad es un caso particular de una medida de credibilidad, y que cumple la propiedad $\operatorname{Nec}(A \cap B)=\operatorname{mín}\{N e c(A), N e c(B)\}$. Una relación detallada entre las medidas difusas anteriormente nombradas, al igual que una clasificación, fue realizada por Banon (1981).

Como se mencionó antes, es díficil desarrollar una teoría general de las medidas difusas sin imponer condiciones adicionales. En este sentido, Wang $(1984,1985)$ introduce algunos conceptos nuevos sobre las características estructurales de las medidas difusas, y por medio de ellos genera un desarrollo de la teoría general de las medidas difusas: nulaaditividad, autocontinuidad, autocontinuidad uniforme, pseudo nulaaditividad y pseudo autocontinuidad, entre otras. Estos conceptos estructurales de las medidas difusas han servido para garantizar condiciones suficientes y necesarias para generalizar teoremas de la teoría clásica de la medida al contexto de las medidas difusas.

Posteriormente, muchos autores se han dedicado a mejorar los resultados propuestos. Por ejemplo, un estudió de los conceptos dados por Wang y la relación existente entre ellos es realizado por Sun (1992); un análisis sobre las condiciones de continuidad y nulaaditividad de las medidas difusas fue hecho por Asahina et al. (2006).

Actualmente, las medidas difusas se han aplicado en varias disciplinas como la inteligencia artificial, psicología, teoría de juegos, teoría de la decisión, economía, redes neuronales, procesamiento de imágenes, entre otras (Liginlal y Terence, 2006; Rama y Tarres, 2007). Para la comunidad científica es una rama de la matemática interesante, donde hay mucho que explorar y que tiene aplicaciones potenciales.

Unido al concepto de medida difusa se encuentra el de integral difusa. Murofushi y Sugeno (2000) definen "la integral difusa" como la integral respecto a una medida difusa. Entre las integrales respecto a una medida difusa se encuentran las integrales dadas por Sugeno y por Weber (Sugeno, 1974; Weber, 1984).

Sugeno introduce la siguiente definición de integral difusa, conocida como integral de Sugeno: Sea $\mu$ una medida difusa sobre $X$ y $f: X \rightarrow[0,1]$ una función medible no negativa con respecto a la medida difusa $\mu$; entonces la integral de $f$ respecto a $\mu$ es dada por:

$$
f f d \mu=\sup _{\alpha \in[0,1]}[\alpha \wedge \mu(\{x \mid f(x) \geq \alpha\})] \text {, }
$$

donde $\wedge$ es el operador mínimo. Es de mencionar, que aunque las medidas difusas son una extensión de las medidas de probabilidad, la integral de Sugeno no es una extensión de la integral de Lebesgue. Si reemplazamos la suma y la multiplicación de los números reales por el supremo y su ínfimo respectivamente en la fórmula de la integral de Lebesgue sobre la recta real, se obtiene la integral de Sugeno. Obsérvese que si $A$ es un conjunto clásico su integral de Sugeno es igual a la medida de $A$; debido a esto, sí la medida utilizada no es aditiva, la integral de Sugeno es la generalización de 
la medida de Lebesgue, pero no de la integral de Lebesgue.

Por otra parte, Weber (1984) introduce otra integral respecto a una medida difusa que llamó la integral de Choquet, y definida por:

$$
f f d \mu=\int_{0}^{\infty} \mu(\{x \mid f(x) \geq \alpha\}) d \alpha .
$$

Dado que varias definiciones para la integral de Choquet son equivalentes a las definiciones para la integral de Lebesgue, puede considerarse que la integral de Choquet es una generalización de la integral de Lebesgue (De Campos y Bolaños, 1992).

Es de mencionar que mientras la integral de Sugeno se basa en operadores no lineales (mínimo y máximo), la integral de Choquet se basa en operadores lineales (suma y producto). La integral de Sugeno tiene como aplicaciones la evaluación subjetiva de fenómenos y la integral de Choquet es utilizada para representar medidas estadísticas como la media, la mediana y L-estimadores.

El propósito de este trabajo es realizar un estudio de las medidas difusas y las integrales difusas, incluyendo principalmente sus propiedades, clasificaciones, ejemplos y propiedades estructurales.

\section{Medidas difusas}

En muchas situaciones reales, a menudo donde hay manipulación de la información nos encontramos con obstáculos debido a la incertidumbre que puede estar dada por la imposibilidad de utilizar datos exactos, la impresición e indecisión de los fenómenos a estudiar, etc. Dicha incerteza no tiene necesariamente que ser aleatoria; en consecuencia esta incertidumbre no tiene que ser medida a través de la probabilidad.

La medida de probabilidad constituye un ejemplo muy importante de medida clásica, pero es aplicable solamente en ciertos casos especiales de incertidumbre basada en la aleatoriedad, la cual no es aplicable a la impresición e incerteza de la información, que son la base del razonamiento humano. Sus limitaciones fueron cada vez más reconocidas.

En 1965 Zadeh abrió las puertas a la solución al problema presentado por la medida de probabilidad dando la definición de conjunto difuso. Para complementar la solución aparecen los términos medida difusa e integral difusa, que fueron introducidos por Michio Sugeno como la forma más adecuada de medir ciertos grados de incerteza, valores que dependen únicamente de la subjetividad humana.

La subjetividad humana se puede clasificar en dos tipos: la incerteza dada por la impresición y la incerteza dada por la indecisión; un conocimiento es impreciso cuando cuenta solamente con predicados vagos, es decir, que las variables no reciben un valor preciso, sino que solamente se especifica un subconjunto al que pertenecen, como: "Carlos es alto", "Diana tiene entre 25 y 30 años"; un conocimiento es indeciso cuando está expresado con predicados precisos, pero donde no puede establecerse el valor de verdad, como por ejemplo: "creo que ...", "es posible que ..."

El primer tipo de incerteza puede ser estudiado usando el concepto de conjunto difuso (medidas de borrosidad); en cuanto al segundo tipo, puede ser estudiado utilizando las medidas difusas.

Una medida (medida clásica) en un espacio medible $(X, \mathscr{A})$, donde $\mathscr{A}$ es una $\sigma$-álgebra, es una aplicación $\mu: \mathscr{A} \rightarrow[0, \infty]$ que satisface las propiedades:

$\left(\mu_{1}\right) \mu(\varnothing)=0$.

$\left(\mu_{2}\right)$ Si $A_{1}, \ldots, A_{n}, \ldots \in \mathscr{A}$ es una familia de conjuntos disjuntos dos a dos, entonces

$$
\mu\left(\bigcup_{n=1}^{\infty} A_{n}\right)=\sum_{n=1}^{\infty} \mu\left(A_{n}\right) .
$$

La segunda propiedad es conocida como la $\sigma$ aditividad, y constituye la principal característica de las medidas clásicas. Aunque dicha propiedad puede ser muy efectiva y conveniente en ciertas aplicaciones, como la estadística y la economía, también puede resultar demasiado inflexible y rígida en otros contextos, como por ejemplo, la inteligencia artificial, las redes neuronales, los procesamientos de imágenes, entre otros (Liginlal y Terence, 2006; Rama y Tarres, 2007), en los cuales es útil definir medidas no aditivas (medidas difusas). Las medidas difusas se caracterizan por la debilitación de la propiedad $\sigma$-aditividad de las 
medidas clásicas, la cual se puede sustituir por una condición más débil, conocida como la monotonía, por lo que también las medidas difusas reciben el nombre de medidas no aditivas.

Algunos conceptos de medida difusa: En 1974 M. Sugeno, en su tesis doctoral, introduce el concepto de medida difusa basándose en el hecho de que para las medidas clásicas la propiedad de $\sigma$ aditividad implica monotonía, continuidad por abajo y continuidad por arriba (Sugeno, 1974).

DEFINICIÓ N 1 Sean $X$ un conjunto clásico y $\mathscr{A}$ una $\sigma$-álgebra de subconjuntos de $X$. Se dice que $\mu: \mathscr{A} \rightarrow[0, \infty]$ es una medida difusa si satisface:

$\left(M_{D_{1}}\right) \mu(\varnothing)=0 \quad$ (condición de frontera).

$\left(M_{D_{2}}\right)$ Para todo $A, B \in \mathscr{A}, A \subseteq B$ se tiene que $\mu(A) \leq \mu(B) \quad$ (monotonía).

En algunos casos es deseable que $\mu$ satisfaga, una o ambas de las siguientes condiciones:

$\left(M_{D_{3}}\right)$ Si $A_{n} \in \mathscr{A}$ y $A_{1} \subseteq A_{2} \subseteq \ldots$, entonces

$$
\mu\left(\bigcup_{n=1}^{\infty} A_{n}\right)=\lim _{n \rightarrow \infty} \mu\left(A_{n}\right)
$$

(continuidad por abajo).

$\left(M_{D_{4}}\right)$ Si $B_{n} \in \mathscr{A}$ y $B_{1} \supseteq B_{2} \supseteq \ldots$ con $\mu\left(B_{1}\right)<\infty$, entonces

$$
\mu\left(\bigcap_{n=1}^{\infty} B_{n}\right)=\lim _{n \rightarrow \infty} \mu\left(B_{n}\right)
$$

(continuidad por arriba).

Una medida difusa que satisface la condición $\left(M_{D_{3}}\right)$ es llamada continua por debajo, y si satisface $\left(M_{D_{4}}\right)$ entonces se dice que es una medida difusa continua por arriba. Si la medida difusa satisface las dos condiciones, entonces se dice que es una medida difusa según Sugeno, o que es una medida difusa continua.
Cuando se desea caracterizar la incertidumbre se utilizan las medidas difusas normalizadas, las cuales surgen al adicionar la condición $\mu(X)=1$.

Cuando $X$ es finito, las condiciones $\left(M_{D 3}\right)$ y $\left(M_{D 4}\right)$ no son necesarias, porque si se tuviera una sucesión creciente o decreciente de una colección finita de conjuntos, tal sucesión será estacionaria.

Ejemplo 1 Sea $X=\{0,1\}, \mathscr{A}=\mathscr{P}(X)$ y $\mu$ definida por

$$
\mu(A)= \begin{cases}0, & \text { si } A=\varnothing \\ 0,5, & \text { si } A=\{0\} \text { o } A=\{1\} \\ 1, & \text { si } A=X\end{cases}
$$

Obsérvese que $\mu$ cumple con $\left(M_{D_{1}}\right)$ y $\left(M_{D_{2}}\right)$. Como $X$ es finito, se cumplen $\left(M_{D_{3}}\right)$ y $\left(M_{D_{4}}\right)$. Entonces $\mu$ es una medida difusa y también es una medida difusa según Sugeno.

En busca de un concepto más amplio para la definición de medida difusa, varios autores Garmendia (2005), Murofushi (1987) y Nguyen y Walter (2006), entre otros, han estudiado una definición de medida difusa en la cual se debilita la definición dada inicialmente por Sugeno, eliminando definitivamente $\mu(X)=1$, que no es esencial en la teoría de Lebesgue; se eliminan también las condiciones $\left(M_{D_{3}}\right)$ y $\left(M_{D_{4}}\right)$, que aunque son esenciales para la teoría Lebesgue, no lo son para la integración respecto a una medida difusa, ya que en este caso se utiliza la integral de Sugeno o de Choquet, donde dichas condiciones no son necesarias, pero sí se necesita que $\mu$ sea monótona. De aquí en adelante se entenderá por medida difusa aquella que satisface sólo las condiciones $\left(M_{D_{1}}\right)$ y $\left(M_{D_{2}}\right)$ de la Definición 1.

Es claro que está noción es más general que la introducida por Sugeno, es decir, todas las medidas difusas según Sugeno son medidas difusas. También es de mencionar, que las medidas clásicas son medidas difusas, pero en cambio, no toda medida difusa es una medida clásica.

A continuación se presenta un ejemplo de una medida difusa que no es una medida difusa según Sugeno, porque no cumple con $\left(M_{D_{4}}\right)$. 
Ejemplo 2 Sea $X=\mathbb{R}, \mathscr{A}=\mathscr{P}(X)$ y $\mu$ : $\mathscr{P}(X) \rightarrow[0,1]$ definida por

$$
\mu(A)= \begin{cases}0, & \text { si } A=\varnothing, \\ 1, & \text { si } A \neq \varnothing .\end{cases}
$$

Nótese que por la definición dada, $\mu$ satisface $\left(M_{D_{1}}\right)$ y $\left(M_{D_{2}}\right)$. En consecuencia $\mu$ es una medida difusa.

$\mu$ no es una medida difusa según Sugeno. En efecto, considérese que $A_{n}=\left(0, \frac{1}{n}\right)$, entonces $\mu\left(\bigcap_{n=1}^{\infty} A_{n}\right)=0$ pero $\lim _{n \rightarrow \infty} \mu\left(A_{n}\right)=1$ ya que $A_{n} \neq \emptyset$ para todo $n=1,2,3, \ldots$ Luego $\mu$ no cumple con $\left(M_{D 4}\right)$.

Otros autores han estudiado el concepto de medida difusa; por ejemplo, Trillas y Alsinas introducen una definición más general de medida difusa, basada en el hecho de que para medir una característica de los elementos de un conjunto $X$, es necesario disponer de una relación de comparación que indique para todo par de elementos si uno presenta más dicha característica que el otro (Trillas y Alsina, 1999; Garmendia, 2001).

Propiedades de las medidas difusas: Una razón importante para el estudio de las medidas difusas en el caso finito, es que hasta ahora casi todas las aplicaciones prácticas se tienen en conjuntos finitos.

Una forma de obtener ejemplos de medidas difusas es partiendo de una medida clásica como lo afirma la siguiente proposición (Geronimo, 1988).

PROPOSICIÓN 1 Sean a una medida clásica y $T:[0, \infty] \rightarrow[0, \infty]$ una función real monótona y creciente tal que $T(0)=0$. Entonces la composición $\mu=T \circ \alpha$ define una medida difusa.

Demostración. En efecto, $\mu(\varnothing)=T \circ \alpha(\varnothing)=$ $T(0)=0$, luego se tiene $\left(M_{D_{1}}\right)$.

Para probar la segunda condición $\left(M_{D_{2}}\right)$, considere $A, B \in \mathscr{A}$ tales que $A \subseteq B$; entonces $\mu(A)=$ $T \circ \alpha(A)=T(\alpha(A))$ y $\mu(B)=T \circ \alpha(B)=T(\alpha(B))$. Como $\alpha(A) \leq \alpha(B)$ y $T$ es creciente, se tiene que $\mu(A) \leq \mu(B)$.
Enseguida daremos dos propiedades generales de las medidas difusas (Geronimo, 1988). En la primera propiedad se demuestra que una medida difusa es un número real no negativo. La segunda propiedad relaciona la medida de la unión y la intersección de dos conjuntos con el máximo y el mínimo de las medidas de estos conjuntos.

Proposición 2 Sea $\mu$ una medida difusa en A; entonces:

(i) $\mu(A) \geq 0, \forall A \in \mathscr{A}$;

(ii) $\forall A, B \in \mathscr{A}, \min \{\mu(A), \mu(B)\} \geq \mu(A \cap B) y$ máx $\{\mu(A), \mu(B)\} \leq \mu(A \cup B)$.

Demostración. (i) Sea $\mathrm{A} \in \mathscr{A}$, como $\varnothing \subseteq A$ y $\mu$ es monótona, entonces $\mu(A) \geq \mu(\varnothing)=0$.

(ii) Como $A \cap B \subseteq A \subseteq A \cup B$ y $A \cap B \subseteq B \subseteq A \cup B$, entonces por $\left(M_{D_{2}}\right)$ se tiene

$$
\mu(A \cap B) \leq \mu(A) \quad \text { y } \quad \mu(A \cap B) \leq \mu(B),
$$

luego $\operatorname{mín}\{\mu(A), \mu(B)\} \geq \mu(A \cap B)$.

Analogámente se realiza la otra parte de la demostración.

Clasificación según la aditividad: En una medida clásica la propiedad de aditividad implica monotonía, pero su recíproco no es cierto. Por ejemplo, sea $X=\{0,1\}$ y $\mu: \mathscr{P}(X) \rightarrow \mathbb{R}^{+}$definida por

$$
\mu(A)= \begin{cases}0, & \text { si } A=\varnothing, \\ 1, & \text { si } A=\{0\} \text { o } A=\{1\}, \\ 3, & \text { si } A=X\end{cases}
$$

Dado que $\{0\} \subseteq X$ y $\mu(\{0\}) \leq \mu(X) ; \mathrm{y}\{1\} \subseteq X$ y $\mu(\{1\}) \leq \mu(X)$, en consecuencia se tiene que $\mu$ es una medida monótona, pero $\mu$ no es una medida aditiva debido a que $\mu(\{0\} \cup\{1\}) \neq \mu(\{0\})+$ $\mu(\{1\})$.

Obsérvese que en este ejemplo $\mu(\{0\} \cup\{1\})=$ $\mu(\{X\})>\mu(\{0\})+\mu(\{1\})$. Pero si se cambia, en la definición de $\mu$, que $\mu(X)=a$, con $a \in(1,2)$, entonces se tendría que $\mu(\{0\} \cup\{1\})=\mu(X)=$ $a<\mu(\{0\})+\mu(\{1\})$. Es decir, se tienen tres posibilidades. La siguiente definición se relaciona con esta observación. 
DEFINICIÓN 2 Una medida difusa $\mu$ definida en $\mathscr{A}$ es llamada:

Aditiva si para todo $A, B \in \mathscr{A} \operatorname{con} A \cap B=\varnothing$ se tiene que $\mu(A \cup B)=\mu(A)+\mu(B)$.

Subaditiva si para todo $A, B \in \mathscr{A}$ se tiene que $\mu(A \cup B) \leq \mu(A)+\mu(B)$.

Superaditiva si para todo $A, B \in \mathscr{A} \operatorname{con} A \cap B=$ $\varnothing$ se tiene que $\mu(A \cup B) \geq \mu(A)+\mu(B)$.

Basado en esta definición se presentan a continuación algunos ejemplos de medidas difusas que se han convertido en fundamento para algunas teorías matemáticas, como la teoría de la probabilidad, la teoría de la evidencia y la teoría de la necesidad, las cuales se pueden clasificar como aditivas, subaditivas y superaditivas, respectivamente.

Ejemplos de medidas difusas aditivas: Un ejemplo importante de medida difusa aditiva es la medida de probabilidad, la cual mide la frecuencia con la que se obtiene un resultado (o conjunto de resultados) al llevar a cabo un experimento aleatorio del que se conocen todos los resultados posibles, bajo condiciones suficientemente estables. La definición formal de medida de probabilidad surge de incluir en la definición de la medida, que $\mu(X)=1$ y la propiedad aditiva (Geronimo, 1988). Formalmente sería:

DEFinición 3 Sea $P$ una función real definida en $\mathscr{A}$. Se dice que $P$ es una medida de probabilidad si se satisface:

$\left(P r_{1}\right) P(A) \geq 0, \quad \forall A \in \mathscr{A}$;

$\left(P r_{2}\right) P(X)=1$

$\left(\mathrm{Pr}_{3}\right)$ si $A_{i}$ es una sucesión en $\mathscr{A}$ tal que $A_{i} \cap A_{j}=$ $\varnothing$ para $i \neq j$, entonces

$$
P\left(\bigcup_{i=1}^{\infty} \mathscr{A}_{i}\right)=\sum_{i=1}^{\infty} P\left(\mathscr{A}_{i}\right) .
$$

Obsérvese que cuando $X$ es un conjunto finito la propiedad $\left(\mathrm{Pr}_{3}\right)$ se puede modificar por

$P(A \cup B)=P(A)+P(B) \forall A, B \in \mathscr{A}$ tales que $A \cap B=\varnothing$.

EjEMPLO 3 Sean $X=\{a, b, c\}, \mathscr{A}=\mathscr{P}(X)$ y $\mu$ una medida definida como:

$$
\mu(A)= \begin{cases}0, & \text { si } \mathrm{A}=\varnothing \\ \frac{1}{3}, & \text { si } A=\{a\},\{b\},\{c\} \\ \frac{2}{3}, & \text { si } A=\{a, b\},\{b, c\},\{a, c\} \\ 1, & \text { si } A=X\end{cases}
$$

Por definición, $\mu$ cumple con $\left(P r_{1}\right)$ y $\left(P r_{2}\right)$. Realizando unos pequeños cálculos se comprueba que $\mu$ cumple con $\left(\mathrm{Pr}_{3}\right)$; por lo tanto, se concluye que $\mu$ es una medida de probabilidad.

A partir de la definición de medida de probabilidad, es posible demostrar ciertas propiedades que ella cumple. Por ejemplo:

PROPOSICIÓN 3 Si P es una medida de probabilidad, entonces satisface las siguientes propiedades:

(i) $P(\varnothing)=0$;

(ii) $\forall A, B \in \mathscr{A}$, si $A \subseteq B$ entonces $P(A) \leq P(B)$.

Consecuentemente, $P$ es una medida difusa.

Demostración. (i) Como $X=X \cup \varnothing$, por $\left(P r_{2}\right)$ y $\left(P_{3}\right)$ se tiene que $P(X)=P(X)+P(\varnothing)$, luego $P(\varnothing)=0$.

(ii) $A \subseteq B$ implica que $B=A \cup(B \backslash A)$; aplicando $\left(P r_{1}\right)$ y $\left(P r_{2}\right)$, se obtiene que

$$
P(B)=P(A)+P(B \backslash A) .
$$

Como $P(B \backslash A) \geq 0$, entonces $P(B) \geq P(A)$.

Como se cumplen estas dos propiedades, entonces se puede concluir que $\mu$ es una medida difusa. 
Ejemplos de medidas difusas subaditivas: Un ejemplo importante de medida difusa subaditiva es la medida de plausibilidad, la cual sirve para medir grados de verosimilitud, los cuales representan la máxima creencia en una hipótesis como resultado de una evidencia (Ayyub y Klir, 2006). La definición formal de medida de plausibilidad es:

DEFINiCión 4 Sean $X$ un conjunto y $\mathscr{A}$ una $\sigma$-álgebra de subconjuntos de $X$. Una medida de plausibilidad es una función $P l: \mathscr{A} \rightarrow[0,1]$ que satisface:

$\left(P l_{1}\right) P l(\varnothing)=0$

$\left(P l_{2}\right) P l(X)=1$

$\left(P l_{3}\right)$ si $\left\{A_{i}\right\}_{i=1}^{n}$ es una colección de elementos de $\mathscr{A}$, entonces

$$
\begin{gathered}
P l\left(A_{1} \cap A_{2} \ldots \cap A_{n}\right) \leq \sum_{j} P l\left(A_{j}\right)-\sum_{j<k} P l\left(A_{j} \cup A_{k}\right) \\
+\ldots+(-1)^{n+1} P l\left(A_{1} \cup A_{2} \ldots \cup A_{n}\right) .
\end{gathered}
$$

Se utiliza $P l$ para nombrar las medidas de plausibilidad; la palabra plausibilidad significa verosimilitud o admisibilidad.

Observe que cuando $n=2$, la propiedad $\left(\mathrm{Pl}_{3}\right)$ se escribe como

$$
P l\left(A_{1} \cap A_{2}\right) \leq P l\left(A_{1}\right)+P l\left(A_{2}\right)-P l\left(A_{1} \cup A_{2}\right) .
$$

En consecuencia,

$$
P l\left(A_{1} \cup A_{2}\right) \leq P l\left(A_{1}\right)+P l\left(A_{2}\right) ;
$$

por lo tanto, se puede concluir que $P l$ es una medida subaditiva.

De la definición de medida de plausibilidad se deduce que

$$
1 \leq P l(A)+P l\left(A^{c}\right) .
$$

En efecto,

$$
1=P l(X)=P l\left(A \cup A^{c}\right) \leq P l(A)+P l\left(A^{c}\right) .
$$

Ejemplos de medidas difusas superaditivas: Un ejemplo importante de medida difusa superaditiva es la medida de credibilidad, la cual es importante en el estudio de la teoría de la evidencia; esta teoría se centra en la credibilidad que se asigna a que un evento pueda ocurrir (o haya ocurrido), desde el punto de vista y de acuerdo con la experiencia de la persona que toma las decisiones, en contraste con la probabilidad clásica, que supone la existencia de valores de probabilidad asociados a eventos determinados independientemente de que el observador pueda conocer el valor real de la probabilidad (Ayyub y Klir, 2006). La definición formal de medida de credibilidad es:

DEFINICIÓn 5 Sean $X$ un conjunto y $\mathscr{A}$ una $\sigma$-álgebra de subconjuntos de $X$. Una medida de credibilidad es una función $\mathrm{Bel}: \mathscr{A} \rightarrow[0,1]$ que satisface:

$\left(B_{1}\right) \operatorname{Bel}(\varnothing)=0$

$\left(B_{2}\right) \operatorname{Bel}(X)=1$

$\left(B_{3}\right)$ si $A_{1}, A_{2}, \ldots, A_{n}$ es una colección de elementos de $\mathscr{A}$, entonces

$$
\begin{aligned}
& \operatorname{Bel}\left(A_{1} \cup A_{2} \ldots \cup A_{n}\right) \geq \\
& \sum_{j} \operatorname{Bel}\left(A_{j}\right)-\sum_{j<k} \operatorname{Bel}\left(A_{j} \cap A_{k}\right) \\
& +\ldots+(-1)^{n+1} \operatorname{Bel}\left(A_{1} \cap A_{2} \ldots \cap A_{n}\right) .
\end{aligned}
$$

Se utiliza Bel para nombrar las medidas de credibilidad, debido a que la palabra proviene del inglés believability, que significa credibilidad.

Observe que cuando $n=2$, la propiedad $\left(B_{3}\right)$ se escribe como

$\operatorname{Bel}\left(A_{1} \cup A_{2}\right) \geq \operatorname{Bel}\left(A_{1}\right)+\operatorname{Bel}\left(A_{2}\right)-\operatorname{Bel}\left(A_{1} \cap A_{2}\right)$.

Además, si $A_{1} \cap A_{2}=\varnothing$, entonces

$$
\operatorname{Bel}\left(A_{1} \cup A_{2}\right) \geq \operatorname{Bel}\left(A_{1}\right)+\operatorname{Bel}\left(A_{2}\right) ;
$$

por lo tanto, se puede concluir que $\mathrm{Bel}$ es una medida superaditiva.

A $\operatorname{Bel}(A)$ se lo llama "grado de creencia", y representa la mínima creencia en la hipótesis $A$ como resultado de una prueba. 
Cuando los conjuntos son disjuntos dos a dos, la propiedad $\left(B_{3}\right)$ se requiere para que los grados de credibilidad asociados con la unión de los conjuntos no sea menor que la suma de los grados de credibilidad asociados a cada conjunto individualmente.

Considérese el siguiente ejemplo:

EJEMPLO 4 Sean $X=\{a, b\}, \mathscr{A}=\mathscr{P}(X)$ y $\mu$ una medida definida por

$$
\mu(A)= \begin{cases}0, & \text { si } A=\varnothing, \\ t, & \text { si } A=\{a\},\{b\}, \quad \text { donde } t \in(0,1), \\ 1, & \text { si } A=X\end{cases}
$$

Se puede verificar que $\mu$ es una medida monótona. Ahora, si $t \in\left(0, \frac{1}{2}\right), \mu$ es una medida de credebilidad. Si $t \in\left(\frac{1}{2}, 1\right), \mu$ es una medida de plausibilidad.

PROPOSICIÓN 4 Si Bel es una medida de credibilidad, entonces satisface las siguientes propiedades:

(i) Bel es una medida difusa monótona en $X$.

(ii) $\operatorname{Bel}(A)+\operatorname{Bel}\left(A^{c}\right) \leq 1$.

Demostración. (i) Por definición $\operatorname{Bel}(\varnothing)=0$. Para demostrar la propiedad de monotonía, considérense $A, B \in \mathscr{A} \operatorname{con} A \subseteq B$ y $C=B-A$.

Entonces, aplicando $\left(B_{3}\right)$ para $n=2$, y el hecho de que $A \cup C=B$, se tiene que

$$
\begin{aligned}
\operatorname{Bel}(B) & =\operatorname{Bel}(A \cup C) \\
& \geq \operatorname{Bel}(A)+\operatorname{Bel}(C)-\operatorname{Bel}(A \cap C) .
\end{aligned}
$$

Puesto que $A \cap C=\varnothing$ y $\operatorname{Bel}(\varnothing)=0$, se concluye que

$$
\operatorname{Bel}(B) \geq \operatorname{Bel}(A)+\operatorname{Bel}(C),
$$

y en consecuencia $\operatorname{Bel}(B) \geq \operatorname{Bel}(A)$.

(ii) Como $A \cap A^{c}=\varnothing$, se tiene que

$$
\begin{aligned}
1=\operatorname{Bel}(X) & =\operatorname{Bel}\left(A \cup A^{c}\right) \\
& \geq \operatorname{Bel}(A)+\operatorname{Bel}\left(A^{c}\right) .
\end{aligned}
$$

Si $\mu$ es una medida difusa sobre $\mathscr{A}$, se puede definir una medida difusa dual dada por

$$
\mu^{d}(A)=\mu(X)-\mu\left(A^{c}\right), \quad \forall A \in \mathscr{A} .
$$

Pero como se está trabajando con medidas difusas normalizadas, es decir, con $\mu(X)=1$, entonces

$$
\mu^{d}(A)=1-\mu\left(A^{c}\right), \quad \forall A \in \mathscr{A} .
$$

En consecuencia, para cada medida de credibilidad se puede definir su medida de plausibilidad por medio de la ecuación $\operatorname{Pl}(A)=1-\operatorname{Bel}\left(A^{c}\right)$. Nótese que a partir de esta ecuación se obtiene que $P l\left(A^{c}\right)=1-\operatorname{Bel}(A), \operatorname{Bel}(A)=1-P l\left(A^{c}\right)$ y $\operatorname{Bel}\left(A^{c}\right)=1-P l(A)$.

$\operatorname{Bel}\left(A^{c}\right)$ se llama "el grado de duda en $A$ ", y representa la mínima creencia en la negación de la hipótesis $A$, como resultado de una prueba.

Como se mencionó, las medidas de credibilidad y las medidas plausibilidad son dos medidas difusas duales, que adicionalmente son la base de una teoría conocida como la Teoría de la evidencia, la cual fue desarrollada por Dempster (1967a, 1967b) y posteriormente extendida por Shafer (1976). Esta teoría es una extensión de la teoría de la probabilidad para representar la ignorancia y para manejar la necesidad de que las creencias asignadas a un evento y su negación sumen uno. Ella se centra en la credibilidad que se asigna a que un evento pueda ocurrir (o haya ocurrido), desde el punto de vista y de acuerdo con la experiencia de la persona que toma las decisiones, en contraste con la probabilidad clásica, que supone la existencia de valores de probabilidad asociados a eventos determinados, independientemente de que el observador pueda conocer el valor real de la probabilidad.

Por otra parte, se puede mostrar fácilmente que las medidas de credibilidad y plausibilidad satisfacen la relación $P l(A) \geq \operatorname{Bel}(A), \forall A \in \mathscr{A}$.

La anterior desigualdad se puede interpretar, para algunas aplicaciones, como que la medida de credibilidad es el límite inferior y la medida de plausibilidad como el límite superior de una prueba sólida. 
Otros dos ejemplos importantes de medidas difusas: Una rama de la teoría de la evidencia es la teoría de la posibilidad, que fue estudiada por Zadeh. Una medida de posibilidad es una medida de plausibilidad a la que se le impone la condición $P l(A \cup B)=\operatorname{máx}\{P l(A), P l(B)\}$.

De forma análoga, cuando a una medida de credibilidad se le impone la condición de que $\operatorname{Bel}(A \cap B)=\min \{\operatorname{Bel}(A), \operatorname{Bel}(B)\}$, se obtiene una medida difusa conocida como medida de necesidad.

Formalmente se tienen las siguientes definiciones (Garmendia, 2001, 2005).

DEFINICIÓN 6 Dado un espacio medible $(X, \mathscr{A})$, una medida de posibilidad es una función $\Pi: \mathscr{A} \rightarrow[0,1]$ que satisface:

$\left(P_{1}\right) \Pi(\varnothing)=0, \quad \Pi(X)=1 ;$

$\left(P_{2}\right) A \subseteq B$ implica que $\Pi(A) \leq \Pi(B) ;$

$\left(P_{3}\right)$ dado un conjunto de índices $I$,

$$
\Pi\left(\bigcup_{i \in I} A_{i}\right)=\sup _{i \in I}\left\{\Pi\left(A_{i}\right)\right\} .
$$

DEFINICIÓN 7 Dado un espacio medible $(X, \mathscr{A})$, una medida de necesidad es una función Nec : $\mathscr{A} \rightarrow[0,1]$ que satisface:

$\left(N_{1}\right) \operatorname{Nec}(\varnothing)=0, \quad \operatorname{Nec}(X)=1 ;$

$\left(N_{2}\right) A \subseteq B \Rightarrow N e c(A) \leq N e c(B)$

$\left(N_{3}\right)$ dado un conjunto de índices $I$,

$$
\operatorname{Nec}\left(\bigcap_{i \in I} A_{i}\right)=\inf _{i \in I}\left\{\operatorname{Nec}\left(A_{i}\right)\right\} \text {. }
$$

Las medidas de posibilidad son medidas difusas normales que cumplen con la propiedad subaditiva, mientras que las medidas de necesidad son medidas difusas normales que cumplen la propiedad superaditiva (Garmendia, 2001, 2005). Obsérvese que a partir de las propiedades se tiene que una medida de necesidad se puede obtener a partir de una medida de posibilidad, por medio de la ecuación

$$
\operatorname{Nec}(A)=1-\Pi\left(A^{c}\right) \text {. }
$$

El Ejemplo 2 satisface las condiciones para ser una medida difusa de posibilidad.

El siguiente resultado da algunas propiedades que cumplen las medidas de posibilidad.

PROPOSICIÓN 5 Dado un espacio medible $(X, \mathscr{A})$, una medida de posibilidad $\Pi: \mathscr{A} \rightarrow[0,1]$ cumple con las siguientes propiedades:

(i) $\operatorname{máx}\left\{\Pi(A), \Pi\left(A^{c}\right)\right\}=1$;

(ii) $\Pi(A)+\Pi\left(A^{c}\right) \geq 1$.

\section{Demostración.}

(i) $\begin{aligned} 1 & =\Pi(X)=\Pi\left(A \cup A^{c}\right) \\ & =\operatorname{máx}\left\{\Pi(A), \Pi\left(A^{c}\right)\right\}\end{aligned}$

(ii) Se tiene que

$$
\begin{aligned}
1 & =\Pi(X)=\Pi\left(A \cup A^{c}\right) \\
& =\operatorname{máx}\left\{\Pi(A), \Pi\left(A^{c}\right)\right\} \\
& \leq \operatorname{máx}\left\{\Pi(A), \Pi\left(A^{c}\right)\right\}+\operatorname{mín}\left\{\Pi(A), \Pi\left(A^{c}\right)\right\} \\
& =\Pi(A)+\Pi\left(A^{c}\right) .
\end{aligned}
$$

En el caso de las medidas de necesidad, se tiene el siguiente resultado análogo.

PROPOSICIÓN 6 Dado un espacio medible $(X, \mathscr{A})$, una medida de necesidad Nec: $\mathscr{A} \rightarrow[0,1]$ cumple con las siguientes propiedades:

(i) $\min \left\{\operatorname{Nec}(A), \operatorname{Nec}\left(A^{c}\right)\right\}=0$;

(ii) $\operatorname{Nec}(A)+\operatorname{Nec}\left(A^{c}\right) \leq 1$.

Demostración.

(i) $0=\operatorname{Nec}(\varnothing)=\operatorname{Nec}\left(A \cap A^{c}\right)$ $=\min \left\{\operatorname{Nec}(A), \operatorname{Nec}\left(A^{c}\right)\right\}$. 
(ii) Se tiene que mín $\left\{\operatorname{Nec}(A), N e c\left(A^{c}\right)\right\}=0$, y además que máx $\left\{\operatorname{Nec}(A), N e c\left(A^{c}\right)\right\} \leq$ $\operatorname{Nec}(X)$; entonces,

$$
\begin{aligned}
& \text { mín }\left\{\operatorname{Nec}(A), \operatorname{Nec}\left(A^{c}\right)\right\} \\
& + \text { máx }\left\{\operatorname{Nec}(A), \operatorname{Nec}\left(A^{c}\right)\right\} \leq \operatorname{Nec}(X),
\end{aligned}
$$

de donde se concluye que

$$
\operatorname{Nec}(A)+\operatorname{Nec}\left(A^{c}\right) \leq 1 .
$$

El siguiente resultado presenta algunas relaciones que existen entre las medidas de posibilidad y la medidas de necesidad.

PROPOSICIÓN 7 Dados un espacio medible $(X, \mathscr{A})$, una medida de posibilidad $\Pi: \mathscr{A} \rightarrow[0,1]$ y una medida de necesidad Nec: $\mathscr{A} \rightarrow[0,1]$, se tienen las siguientes relaciones:

(i) $\operatorname{sinec}(A) \geq 0$, entonces $\Pi(A)=1$;

(ii) si $\Pi(A) \leq 1$, entonces $N e c(A)=0$.

\section{Demostración.}

(i) Se sabe que mín $\left\{\operatorname{Nec}(A), \operatorname{Nec}\left(A^{c}\right)\right\}=0$, y por hipótesis se tiene que $\operatorname{Nec}(A) \geq 0$; entonces $\mathrm{Nec}\left(A^{c}\right)=0$. Pero por la ecuación (1) se tiene que $\operatorname{Nec}\left(A^{c}\right)=1-\Pi(A)$, de donde se concluye que $1-\Pi(A)=0$, y en consecuencia $\Pi(A)=1$.

(ii) Sabemos que máx $\left\{\Pi(A), \Pi\left(A^{c}\right)\right\}=1$, y por hipótesis se tiene que $\Pi(A) \leq 1$; entonces $\Pi\left(A^{c}\right)=1$. Pero por la ecuación (1) se tiene que $\Pi\left(A^{c}\right)=1-\operatorname{Nec}(A)$, por lo tanto $1-\operatorname{Nec}(A)=1$, de donde se concluye que $\operatorname{Nec}(A)=0$.

Las $\lambda$-medidas de Sugeno: En 1974, M. Sugeno introduce el concepto de $\lambda$-medida cuya característica es que es una medida normal que posee la propiedad de $\lambda$-aditividad (condición $\left(\lambda_{2}\right)$ de la siguiente definición). Es de resaltar que las $\lambda$ medidas también son medidas difusas.
Definición 8 Sean $\lambda \in(-1, \infty)$ y $(X, \mathscr{A})$ un espacio medible. Una $\lambda$-medida es una función $g_{\lambda}: \mathscr{A} \rightarrow[0,1]$ que satisface:

$\left(\lambda_{1}\right) g_{\lambda}(X)=1$

$\left(\lambda_{2}\right) \forall A, B \in \mathscr{A}$, con $A \cap B=\varnothing$, se tiene que

$g_{\lambda}(A \cup B)=g_{\lambda}(A)+g_{\lambda}(B)+\lambda \cdot g_{\lambda}(A) \cdot g_{\lambda}(B)$.

Obsérvese que la primera propiedad garantiza que $g_{\lambda}$ es normal; ahora, la segunda propiedad hace, dependiendo del valor de $\lambda$, que se cumpla o no la propiedad de aditividad: si $\lambda$ es negativa, $g_{\lambda}$ es subaditiva; si $\lambda$ es positiva, $g_{\lambda}$ es superaditiva; y si $\lambda=0, g_{\lambda}$ es aditiva. La siguiente proposición presenta algunas propiedades de una $\lambda$ medida (Geronimo, 1988; Nguyen y Walter, 2006).

PROPOSICIÓN 8 Si g $g_{\lambda}$ es una $\lambda$-medida con $\lambda>-1$, entonces se cumplen las siguientes propiedades:

(i) $g_{\lambda}$ es una medida difusa;

(ii) $\forall A, B \in \mathscr{A}$ se tiene que

$$
\begin{aligned}
& g_{\lambda}(A \cup B)= \\
& \frac{g_{\lambda}(A)+g_{\lambda}(B)-g_{\lambda}(A \cap B)+\lambda \cdot g_{\lambda}(A) \cdot g_{\lambda}(B)}{1+\lambda \cdot g_{\lambda}(A \cap B)} ;
\end{aligned}
$$

(iii) $g_{\lambda}(A)+g_{\lambda}\left(A^{c}\right)=1-\lambda \cdot g_{\lambda}(A) \cdot g_{\lambda}\left(A^{c}\right)$.

Demostración. (i) Como $g_{\lambda}$ es una $\lambda$-medida, entonces se tiene que $g_{\lambda}(X)=1$. Por otra parte, $X=X \cup \varnothing$, luego

$$
\begin{aligned}
1 & =g_{\lambda}(X \cup \varnothing) \\
& =g_{\lambda}(X)+g_{\lambda}(\varnothing)+\lambda \cdot g_{\lambda}(X) \cdot g_{\lambda}(\varnothing) \\
& =1+g_{\lambda}(\varnothing)+\lambda \cdot g_{\lambda}(\varnothing) \\
& =1+g_{\lambda}(\varnothing)(1+\lambda) ;
\end{aligned}
$$

en consecuencia, $g_{\lambda}(\varnothing)(1+\lambda)=0$, así que $g_{\lambda}(\varnothing)=0$. Luego se cumple $\left(M_{D_{1}}\right)$.

Para la segunda propiedad $\left(M_{D_{2}}\right)$ consideremos que $A \subseteq B$. Entonces existe $C \in \mathscr{A}$ tal que $B=A \cup C$, luego

$$
\begin{aligned}
g_{\lambda}(B) & =g_{\lambda}(A \cup C) \\
& =g_{\lambda}(A)+g_{\lambda}(C)+\lambda \cdot g_{\lambda}(A) \cdot g_{\lambda}(C) .
\end{aligned}
$$

Pero 


$$
\begin{aligned}
g_{\lambda}(C)+ & \lambda \cdot g_{\lambda}(A) \cdot g_{\lambda}(C) \\
& =g_{\lambda}(C)\left(1+\lambda \cdot g_{\lambda}(A)\right) \geq 0,
\end{aligned}
$$

puesto que $\lambda>-1$ y $0 \leq g_{\lambda}(A) \leq 1$. Así, $g_{\lambda}(A) \leq g_{\lambda}(B)$.

Por lo anterior se concluye que una $\lambda$ medida es una medida difusa.

(ii) Obsérvese que $A \cup B=\left(A \cap B^{c}\right) \cup B$ y $\left(A \cap B^{c}\right) \cap B=\varnothing, \forall A, B \in \mathscr{A}$. Ahora bien,

$$
\begin{aligned}
g_{\lambda}(A \cup B)= & g_{\lambda}\left(A \cap B^{c}\right)+g_{\lambda}(B) \\
& +\lambda \cdot g_{\lambda}\left(A \cap B^{c}\right) \cdot g_{\lambda}(B) .
\end{aligned}
$$

También se tiene que $A=(A \cap B) \cup\left(A \cap B^{c}\right)$ y son disjuntos para cualquier $A, B \in \mathscr{A}$; por lo tanto,

$$
\begin{aligned}
& g_{\lambda}\left(A \cap B^{c}\right)+g_{\lambda}(A \cap B) \\
& +\lambda \cdot g_{\lambda}\left(A \cap B^{c}\right) \cdot g_{\lambda}(A \cap B)=g_{\lambda}(A) .
\end{aligned}
$$

Esto implica que

$$
g_{\lambda}\left(A \cap B^{c}\right)=\frac{g_{\lambda}(A)-g_{\lambda}(A \cap B)}{1+\lambda g_{\lambda}(A \cap B)} .
$$

Por lo tanto,

$$
\begin{gathered}
g_{\lambda}(A \cup B)=\frac{g_{\lambda}(A)-g_{\lambda}(A \cap B)}{1+\lambda g_{\lambda}(A \cap B)}+g_{\lambda}(B) \\
+\lambda \cdot \frac{g_{\lambda}(A)-g_{\lambda}(A \cap B)}{1+\lambda g_{\lambda}(A \cap B)} \cdot g_{\lambda}(B) .
\end{gathered}
$$

Si sumamos la expresión del lado derecho se tiene que el numerador es

$$
\begin{gathered}
g_{\lambda}(A)-g_{\lambda}(A \cap B)+g_{\lambda}(B)+\lambda g_{\lambda}(A \cap B) \cdot g_{\lambda}(B) \\
+\lambda \cdot g_{\lambda}(A) \cdot g_{\lambda}(B)-\lambda \cdot g_{\lambda}(A \cap B) \cdot g_{\lambda}(B),
\end{gathered}
$$

que al simplificarlo queda como

$$
g_{\lambda}(A)+g_{\lambda}(B)-g_{\lambda}(A \cap B)+\lambda \cdot g_{\lambda}(A) \cdot g_{\lambda}(B),
$$

y en consecuencia se obtiene la ecuación en (ii). (iii) Como $X=A \cup A^{c}$ y $A \cap A^{c}=\varnothing$, se tiene que

$$
\begin{aligned}
1 & =g_{\lambda}(X)=g_{\lambda}\left(A \cup A^{c}\right) \\
& =g_{\lambda}(A)+g_{\lambda}\left(A^{c}\right)+\lambda \cdot g_{\lambda}(A) \cdot g_{\lambda}\left(A^{c}\right) .
\end{aligned}
$$

De donde se concluye que

$$
g_{\lambda}(A)+g_{\lambda}\left(A^{c}\right)=1-\lambda \cdot g_{\lambda}(A) \cdot g_{\lambda}\left(A^{c}\right) .
$$

El proceso de construir una $\lambda$-medida en una $\sigma$-álgebra tiene mucha importancia y significado práctico. Si $X=\left\{x_{1}, x_{2}, \ldots, x_{n}\right\}$ es un conjunto finito, $\mathscr{C}$ está constituido por todos los conjuntos unitarios de $X$, se conoce la medida de cada elemento $x_{i}\left(g_{i}=g_{\lambda}\left(x_{i}\right)\right), i=1,2, \ldots n$, con $0 \leq g_{i}<g_{\lambda}(X)<\infty$, y existen al menos dos puntos $x_{j}$ donde $g_{j}>0$, entonces $g_{\lambda}$ define una $\lambda$-medida a partir de $\mathscr{C}$ con parámetro $\lambda$. Si $g_{\lambda}(X)=\sum_{i=1}^{n} g_{i}$, entonces $\lambda=0$; por otro lado, si $g_{\lambda}(X) \neq \sum_{i=1}^{n} g_{i}$, entonces $\lambda$ se puede encontrar por medio de la ecuación (Wang y Klir, 2009)

$$
1+\lambda g_{\lambda} \cdot(X)=\prod_{i=1}^{n}\left(1+\lambda \cdot g_{i}\right) \text {. }
$$

EJEMPLO 5 Un profesor de matemáticas evalúa a sus estudiantes de acuerdo con las siguientes materias: geometría, álgebra y estadística. El docente asigna los grados de importancia a las materias como sigue: geometría el $40 \%$, estadística el $40 \%$ y álgebra el $50 \%$.

Si $x_{1}$ representa geometría, $x_{2}$ representa estadística y $x_{3}$ representa álgebra, entonces se tiene que el grado de importancia de las materias satisface: $g_{1}=g_{\lambda}\left(\left\{x_{1}\right\}\right)=0,4, g_{2}=g_{\lambda}\left(\left\{x_{2}\right\}\right)=0,4 \mathrm{y}$ $g_{3}=g_{\lambda}\left(\left\{x_{3}\right\}\right)=0,5$.

Para encontrar el grado de importancia entre la relación de las materias se debe encontrar el valor de $\lambda$, para lo cual se debe utilizar la ecuación (3) y el hecho de que la $\lambda$-medida debe ser normal, es decir, que $g_{\lambda}\left(\left\{x_{1}, x_{2}, x_{3}\right\}\right)=g_{\lambda}(X)=1$ :

$$
1+\lambda \mu(X)=\prod_{i=1}^{n}\left(1+\lambda \cdot \mu\left(\left\{x_{i}\right\}\right)\right),
$$

lo cual implica que

$$
1+\lambda=(0,4 \lambda+1)(0,4 \lambda+1)(0,5 \lambda+1),
$$


de donde se llega a la ecuación cúbica

$$
0,08 \lambda^{3}+0,56 \lambda^{2}+0,3 \lambda=0
$$

Las raíces de la anterior ecuación son: 0, -0,584 y $-6,415$. Dado que $\lambda \in(-1, \infty)$ y que el valor de $\lambda=0$ genera una medida aditiva, entonces se debe considerar el valor de $\lambda=-0,584$.

En este caso se tiene que el grado de importancia al interrelacionar las materias es:

$$
\begin{aligned}
g_{\lambda}\left(\left\{x_{1}, x_{2}\right\}\right)= & g_{\lambda}\left(\left\{x_{1}\right\}\right)+g_{\lambda}\left(\left\{x_{2}\right\}\right) \\
& +\lambda \cdot g_{\lambda}\left(\left\{x_{1}\right\}\right) \cdot g_{\lambda}\left(\left\{x_{2}\right\}\right)=0,706 \\
g_{\lambda}\left(\left\{x_{1}, x_{3}\right\}\right)= & g_{\lambda}\left(\left\{x_{1}\right\}\right)+g_{\lambda}\left(\left\{x_{3}\right\}\right) \\
& +\lambda \cdot g_{\lambda}\left(\left\{x_{1}\right\}\right) \cdot g_{\lambda}\left(\left\{x_{3}\right\}\right)=0,78 \\
g_{\lambda}\left(\left\{x_{2}, x_{3}\right\}\right)= & g_{\lambda}\left(\left\{x_{2}\right\}\right)+g_{\lambda}\left(\left\{x_{3}\right\}\right) \\
& +\lambda \cdot g_{\lambda}\left(\left\{x_{2}\right\}\right) \cdot g_{\lambda}\left(\left\{x_{3}\right\}\right)=0,78 \\
g_{\lambda}(X)= & 1 .
\end{aligned}
$$

Como puede observarse de la definición de $g_{\lambda}$, cuando $\lambda=0$ la medida obtenida es una medida difusa aditiva; cuando $\lambda>0$ la medida es superaditiva; $y$ cuando $\lambda<0$ la medida difusa obtenida es subaditiva. Estas relaciones entre las anteriores medidas difusas ya fueron estudiadas (Banon, 1981). Por otra parte, en las secciones anteriores se presentaron dos ejemplos importantes de medidas subaditivas: las medidas de plausibilidad y las medidas de posibilidad; dos ejemplos de medidas superaditivas: las medidas de credibilidad y las medidas de necesidad; así como un ejemplo de una medida aditiva: medida de probabilidad. La (Figura 1) presenta un diagrama que muestra la relación entre $\lambda$-medidas y medidas aditivas, subaditivas y superaditivas.

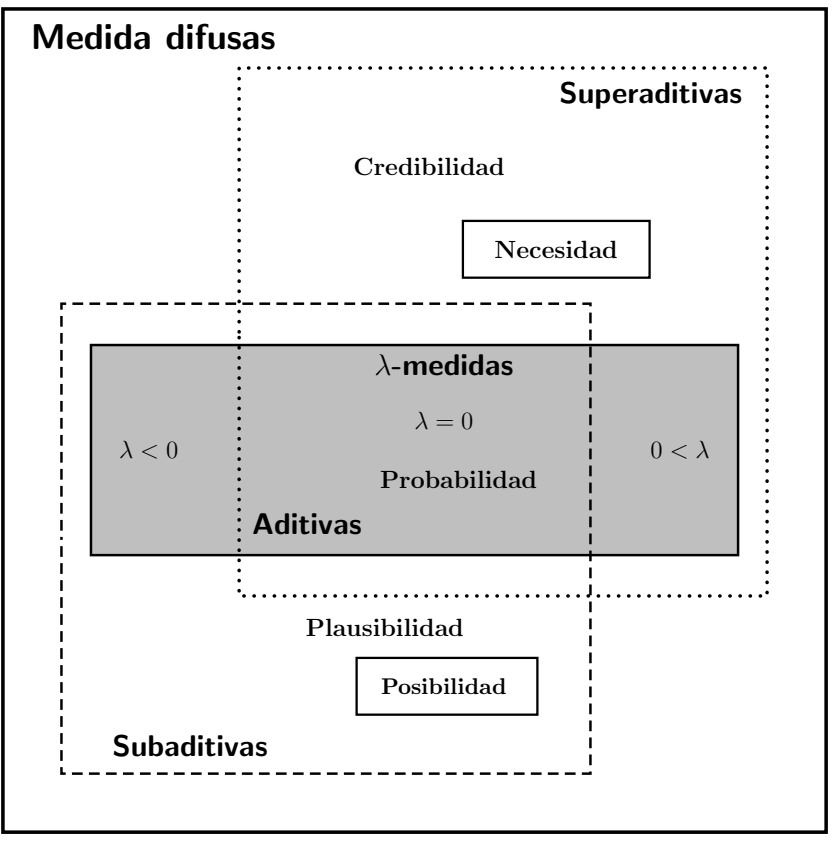

Fig. 1. Relación entre algunas medidas difusas.

\section{Integración respecto a medidas difusas}

Dada una medida difusa, lo inmediatamente natural es considerar la integral con respecto a dicha medida. Estas integrales pueden ser denominadas integrales difusas. El propósito a continuación es presentar dos tipos de integrales difusas: integral de Sugeno e integral de Choquet. Estos dos funcionales se pueden definir sobre cualquier medida difusa. Se presentan, además de sus definiciones, las propiedades más destacadas y conocidas, y algunos resultados análogos a los teoremas de convergencia de la teoría de la medida clásica; posteriormente, haciendo uso del concepto de funciones equiordenadas, se presenta un estudio comparativo entre dichas integrales, con el fin de señalar sus semejanzas y sus diferencias conceptuales.

Integral de Sugeno: A no ser que se diga lo contrario, $(X, \mathscr{A})$ es un espacio de medida, donde $X \in \mathscr{A}, \mu: \mathscr{A} \rightarrow[0, \infty]$ es una medida difusa continua y $\mathscr{G}$ es la clase de todas las funciones medibles no negativas finitas definidas en $(X, \mathscr{A})$ (se dice que una aplicación $f:\left(X, \mathscr{A}_{1}\right) \rightarrow\left(Y, \mathscr{A}_{2}\right)$ entre espacios medibles, es una función medible, si $f^{-1}(B) \in \mathscr{A}_{1}$ para todo $B \in \mathscr{A}_{2}$, es decir $f^{-1}\left(\mathscr{A}_{2}\right) \subset \mathscr{A}_{1}$ (Ash, 2000; Folland, 1999). Dada 
cualquier función $f \in \mathscr{G},[f]^{\alpha}=\{x \mid f(x) \geq \alpha\}$ $\mathrm{y}[f]^{\alpha+}=\{x \mid f(x)>\alpha\}$, donde $\alpha \in(0, \infty]$. Estos conjuntos son llamados $\alpha$-niveles y $\alpha$-niveles estrictos de $f$, respectivamente. El soporte de $f$ es $[f]^{0}=\overline{\{x \mid f(x)>0\}}=\overline{[f]^{0+}}($ Figura 2).

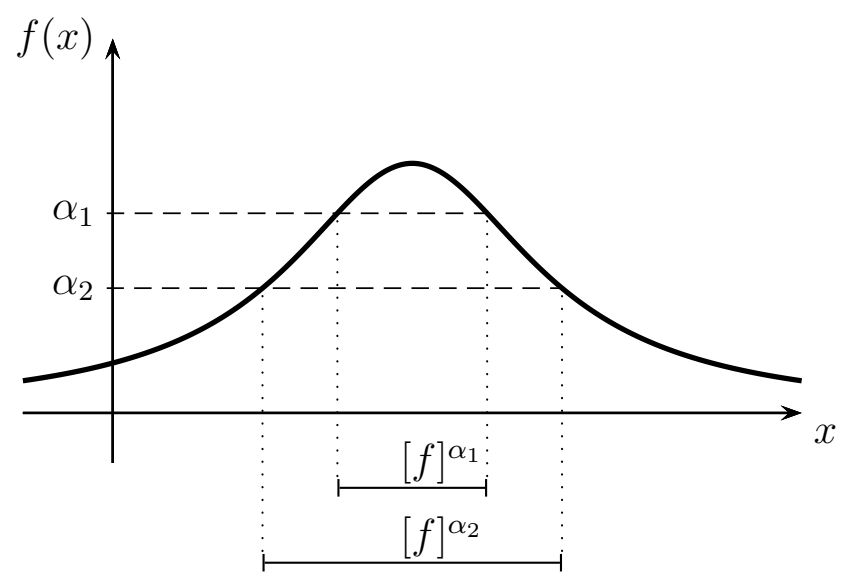

Fig. 2. Los $\alpha$-niveles de $f$.

Dado que el recorrido de las funciones que se considerarán es $[0, \infty)$, se utiliza la siguiente convención: ínf $\operatorname{i\in \varnothing }_{x \in \varnothing} f(x)=\infty$.

Los operadores mínimo y máximo se denotaran por los símbolos $\wedge \mathrm{y} \vee$, respectivamente.

Definición 9 Sea $A \in \mathscr{A}$ y $f \in \mathscr{G}$. La integral de Sugeno de $f$ sobre $A$, con respecto a $\mu$, que se denota por $f_{A} f d \mu$, se define como

$$
f_{A} f d \mu=\sup _{\alpha \in[0, \infty]}\left\{\alpha \wedge \mu\left(A \cap[f]^{\alpha}\right)\right\} \text {. }
$$

Cuando $A=X$, la integral de Sugeno también puede ser denotada por $f f d \mu$.

Cuando se utilice el símbolo $f_{A} f d \mu$, se sobreentenderá que $A \in \mathscr{A}$ y $f \in \mathscr{G}$.

OBSERVACIÓn 1 Si $X=(-\infty, \infty), \mathscr{A}$ es la $\sigma$-álgebra de Borel $\mathscr{B}, \mu$ es la medida de Lebesgue y $f: X \rightarrow[0, \infty)$ es una función de una variable, entonces el significado geométrico de $f_{A} f d \mu$ es la longitud del lado del cuadrado más grande que puede inscribirse entre la curva $f(x)$ y el eje $x$; esto ocurre, dado que el $\sup _{\alpha \in[0, \infty]}\left\{\alpha \wedge \mu\left(A \cap[f]^{\alpha}\right)\right\}$ se obtiene cuando $\alpha=\mu\left(A \cap[f]^{\alpha}\right)$ (Figura 3).

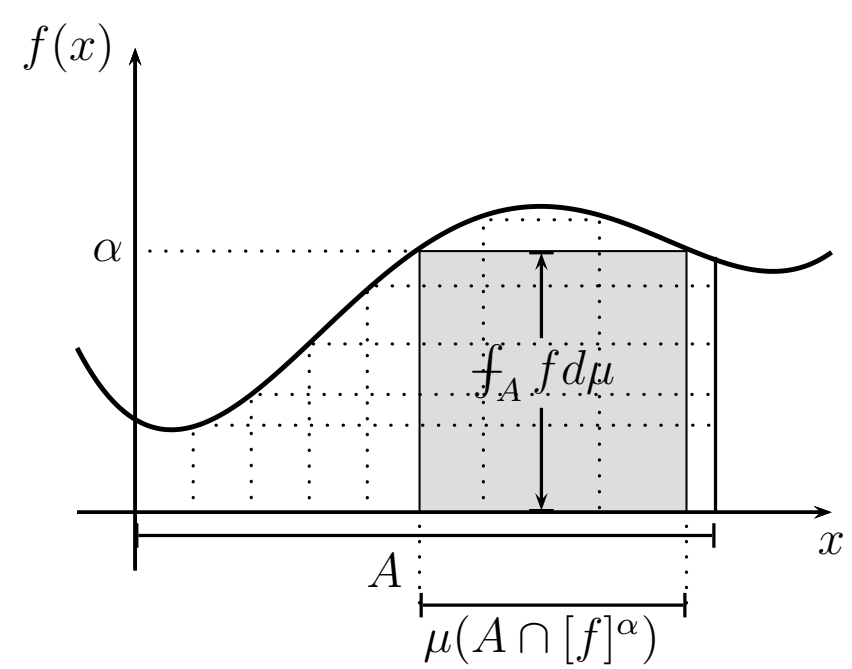

Fig. 3. Intepretación de la integral de Sugeno.

EjEMPLO 6 Sean $X=[0,1], \mathscr{A}$ la clase de todos los conjuntos de Borel en $X$ y $\mu$ la medida de Lebesgue. Si $f(x)=x^{2}$, entonces

$$
f f d \mu=f x^{2} d \mu=\frac{3-\sqrt{5}}{2} .
$$

Nótese que para encontrar el lado del cuadrado de mayor área que se puede inscribir en la región acotada por $f(x)=x^{2}$, el eje $x$ y la recta $x=1$, se debe resolver la ecuación $\alpha=1-\sqrt{\alpha}$, donde $\alpha$ es la altura del cuadrado y $1-\sqrt{\alpha}$ es la medida de la base del cuadrado; aunque dicha ecuación tiene dos soluciones, se ha considerado solo la que pertenece al intervalo $[0,1]$.

Utilizando las definiciones de $[f]^{\alpha},[f]^{\alpha+} \mathrm{y}$ $f_{A} f d \mu$, se pueden demostrar las siguientes igualdades (Wang y Klir, 2009, 1992):

TEOREMA 1 Si $\sigma(f)$ es la $\sigma$-álgebra más pequeña generada por $f$, se tienen que:

$$
\begin{aligned}
f_{A} f d \mu & =\sup _{\alpha \in[0, \infty)}\left\{\alpha \wedge \mu\left(A \cap[f]^{\alpha}\right)\right\} \\
& =\sup _{\alpha \in[0, \infty]}\left\{\alpha \wedge \mu\left(A \cap[f]^{\alpha+}\right)\right\} \\
& =\sup _{\alpha \in[0, \infty)}\left\{\alpha \wedge \mu\left(A \cap[f]^{\alpha+}\right)\right\} \\
& =\sup _{E \in \sigma(f)}\left\{\left(\inf _{x \in E} f(x)\right) \wedge \mu(A \cap E)\right\}
\end{aligned}
$$




$$
=\sup _{E \in \mathscr{A}}\left\{\left(\inf _{x \in E} f(x)\right) \wedge \mu(A \cap E)\right\} .
$$

Propiedades de la integral de Sugeno: A continuación se presentan algunas propiedades de la integral de Sugeno (Wang y Klir, 2009; RománFlores et al., 2007).

Teorema 2 Sean $A, B \in \mathscr{A}, f, g \in \mathscr{G}, \chi_{A}$ es la función característica de $A$ y $k$ cualquier constante en $[0, \infty)$. La $f_{A} f d \mu$ satisface las siguientes propiedades:

1. Si $\mu(A)=0$, entonces $f_{A} f d \mu=0$.

2. Si $\mu$ es continua por abajo $y f_{A} f d \mu=0$, entonces $\mu(A \cap\{x \mid f(x)>0\})=0$.

3. Si $f \leq g$, entonces $f_{A} f d \mu \leq f_{A} g d \mu$.

4. $f_{A} f d \mu=f_{A} f \cdot \chi_{A} d \mu$.

5. $f_{A} k d \mu=k \wedge \mu(A)$.

6. $f_{A}(f+k) d \mu \leq f_{A} f d \mu+f_{A} k d \mu$ $(k \in[0, \infty))$.

7. Si $A \subset B$ entonces $f_{A} f d \mu \leq f_{B} f d \mu$.

8. $f_{A}(f \vee g) d \mu \geq f_{A} f d \mu \vee f_{A} g d \mu$.

9. $f_{A}(f \wedge g) d \mu \leq f_{A} f d \mu \wedge f_{A} g d \mu$.

10. $f_{A \cup B} f d \mu \geq f_{A} f d \mu \vee f_{B} f d \mu$.

11. $f_{A \cap B} f d \mu \leq f_{A} f d \mu \wedge f_{B} f d \mu$.

Las propiedades 1, 3, 4 y 5 se siguen de la definición de integral de Sugeno; la demostración de la propiedad 2 se sigue de la continuidad por abajo; para demostrar la propiedad 6 se debe utilizar el Teorema 1; la propiedad 7 se sigue de las propiedades 3 y 4 ; las propiedades 8 y 9 se siguen de la propiedad 3; las propiedades 10 y 11 se siguen directamente de la propiedad 7.
Aunque algunas de las propiedades presentadas son similares a las de la integral de Lebesgue clásica, otras no, como es el caso de las propiedades 5 y 6, que están relacionadas con la linealidad. Esto implica que la integral de Sugeno carece de dicha propiedad, que sí satisface la integral de Lebesgue. Veamos.

EJEMPLO 7 Sean $X=\{a, b\}, \mathscr{A}=\mathscr{P}(X)$ y

$$
\mu(A)= \begin{cases}0 & \text { si } A=\varnothing \\ 1 & \text { en otros casos. }\end{cases}
$$

Considérese las funciones

$$
f(x)=\left\{\begin{array}{lll}
0 & \text { si } & x=a \\
1 & \text { si } & x=b
\end{array}\right.
$$

y

$$
g(x)=\left\{\begin{array}{lll}
0 & \text { si } & x=b \\
1 & \text { si } & x=a
\end{array}\right.
$$

Aplicando la definición de integral de Sugeno, se tiene que:

$f f d \mu=1, \quad f g d \mu=1 \quad y \quad f(f+g) d \mu=1$.

En conclusión, $f(f+g) d \mu \neq f f d \mu+f g d \mu$.

Ejemplo 8 Sean $X=[0,1], \mathscr{A}$ la clase de todos los conjuntos de Borel en $X$ y $\mu$ la medida de Lebesgue. Si $f(x)=x^{2}$ y $a=2$, al utilizar la Observación 1 se tiene que

$$
\text { faf } d \mu=f 2 x^{2} d \mu=\frac{1}{2}
$$

y

$$
a f f d \mu=2 f x^{2} d \mu=2 \times \frac{3-\sqrt{5}}{2}=3-\sqrt{5} .
$$

Por lo tanto, se puede concluir que $f$ af $d \mu \neq$ af $f d \mu$.

Las siguientes propiedades de la integral de Sugeno son bien conocidas (Murofushi y Sugeno, 2000; Wang y Klir, 2009; Román-Flores et al., 2007).

LEMA 1 Sean $A \in \mathscr{A}$ y $f, g \in \mathscr{G}$. Entonces se tienen las siguientes propiedades. 
1. $f_{A} f d \mu \leq \mu(A)$.

2. Para cualquier $\beta<\alpha$, si $\alpha \leq \mu\left(A \cap[f]^{\beta}\right)$, entonces $\alpha \leq f_{A} f d \mu$.

3. Para cualquier $\beta<\alpha$, si $\alpha \geq \mu\left(A \cap[f]^{\beta}\right)$, entonces $\alpha \geq f_{A} f d \mu$.

4. $f_{A} f d \mu<\alpha$ si y sólo si existe $\beta<\alpha$ tal que $\mu\left(A \cap[f]^{\beta}\right)<\alpha$.

5. $f_{A} f d \mu>\alpha$ si y sólo si existe $\beta<\alpha$ tal que $\mu\left(A \cap[f]^{\beta}\right)>\alpha$.

6. Si $\mu(A)<\infty$, entonces $\alpha \leq f_{A} f d \mu$ si y sólo si $\alpha \leq \mu\left(A \cap[f]^{\alpha}\right)$.

Sean $A \in \mathscr{A}$ y $P$ una proposición con respecto a los puntos de $A$. Si existe $E \in \mathscr{A}$ con $\mu(E)=0$ tal que $P$ es cierto sobre $A \backslash E$, entonces se dice que " $P$ es cierto en casi todas partes de $A$ ". Se abreviará "en casi todas partes" por "c.t.p.”. Si " $g$ es igual a $f$ c.t.p.”, se denotará por “ $g=f$ c.t.p.”.

Un resultado de la teoría clásica de funciones medibles es que si $g=f$ c.t.p., entonces sus integrales son iguales; este resultado no siempre se cumple en el caso de medidas difusas. Por ejemplo, $\operatorname{sean} X=\{a, b\}, \mathscr{A}=P(X)$ y

$$
\mu(A)=\left\{\begin{array}{lll}
1 & \text { si } & A=X \\
0 & \text { si } & A \neq X
\end{array}\right.
$$

Si $g(x)=\left\{\begin{array}{lll}1 & \text { si } & x=a, \\ 0 & \text { si } & x=b,\end{array} \quad\right.$ y $f(x)=1$, entonces, claramente bajo la medida difusa $\mu, g=f$ c.t.p., pero obsérvese que

$$
f g d \mu=0 \quad \text { y } \quad f f d \mu=1 .
$$

Para que el resultado mencionado se cumpla es necesario poner la condición de nulaaditividad sobre la medida difusa. Una función $\mu: \mathscr{A} \rightarrow[0, \infty]$ se denomina nulaaditiva si $\mu(A \cup B)=\mu(A)$, siempre que $A, B \in \mathscr{A}, A \cap B=\varnothing$ y $\mu(B)=0$ (Song y Li, 2005; Wang y Klir, 2009).
TEOREMA $3 f f d \mu=f g d \mu$ cuando $f=g$ c.t.p. si y sólo si $\mu$ es una medida difusa nulaaditiva.

Demostración. Suficiencia: Si $\mu$ es una medida nulaaditiva, entonces $\mu(\{x \mid f(x) \neq g(x)\})=0$. Por otra parte, de la definición de nulaaditividad se sabe que

$$
\begin{aligned}
\mu\left([g]^{\alpha}\right) & \leq \mu\left([f]^{\alpha} \cup\{x \mid f(x) \neq g(x)\}\right) \\
& =\mu\left([f]^{\alpha}\right),
\end{aligned}
$$

para todo $\alpha \in[0, \infty]$. El recíproco de la desigualdad se obtiene de forma equivalente. Así, se puede concluir que $\mu\left([g]^{\alpha}\right)=\mu\left([f]^{\alpha}\right)$ para todo $\alpha \in[0, \infty] \mathrm{y}$, por tanto, de la definición de integral de Sugeno se tiene que

$$
f f d \mu=f g d \mu \text {. }
$$

Necesidad: Para todo $A, B \in \mathscr{A}$ con $\mu(B)=0$, si $\mu(A)=\infty$, entonces, por la monotonía de $\mu$, $\mu(A \cup B)=\infty=\mu(A)$. Ahora, supóngase que $\mu(A)<\infty$ y muéstrese por contradicción que $\mu(A \cup B)=\mu(A)$. Supóngase que estas medidas son diferentes, esto es $\mu(A \cup B)>\mu(A)$; supóngase que $t_{0} \in(\mu(A), \mu(A \cup B))$, y considérense las funciones, iguales en casi todas partes, definidas por

$$
f(x)=\left\{\begin{array}{lll}
t_{0} & \text { si } & x \in A, \\
0 & \text { si } & x \notin A,
\end{array}\right.
$$

y

$$
g(x)=\left\{\begin{array}{lll}
t_{0} & \text { si } & x \in A \cup B \\
0 & \text { si } & x \notin A \cup B .
\end{array}\right.
$$

Debido a que

$$
\mu(\{x \mid f(x) \neq g(x)\})=\mu(B \backslash A) \leq \mu(B)=0,
$$

se tiene que $f=g$ c.t.p.

Así, se debe considerar que

$$
f f d \mu=f g d \mu \text {. }
$$

Ahora, el lado izquierdo es igual a

$$
f f d \mu=t_{0} \wedge \mu(A)=\mu(A),
$$

y el lado derecho es igual a

$$
f g d \mu=t_{0} \wedge \mu(A \cup B)=t_{0} \neq \mu(A) .
$$

Por tanto, se tiene una contradicción. 
Si $\mu$ es nulaaditiva, se puede utilizar el hecho de que $f=g$ c.t.p. sobre $A$, para concluir que $f \cdot \chi_{A}=g \cdot \chi_{A}$ c.t.p. sobre $A$; esto, junto con los teoremas 2 y 3 , lleva al siguiente resultado (Wang y Klir, 2009, 1992).

COROLARIO 1 Si $\mu$ es nulaaditiva, entonces $f_{A} f d \mu=f_{A} g d \mu$ siempre que $f=g$ c.t.p. sobre $A$.

Ahora, si $A, B \in \mathscr{A}$ con $\mu(B)=0$ y $\mu$ es nulaaditiva, entonces se puede utilizar el hecho de que $f \cdot \chi_{A \cup B}=f \cdot \chi_{A}$ c.t.p., para demostrar:

COROLARIO 2 Si $\mu$ es nulaaditiva, entonces para cualquier $f \in \mathscr{G}$,

$$
f_{A \cup B} f d \mu=f_{A} f d \mu
$$

cuando $A, B \in \mathscr{A}$ con $\mu(B)=0$ (Wang y Klir, 2009, 1992).

Integral de Choquet: Debido a que las medidas difusas son una generalización de las medidas de Lebesgue, la integral de Choquet, que se realiza con respecto a una medida difusa, puede considerarse como una extensión natural de la integral de Lebesgue. La integral de Choquet es utilizada con éxito en muchos problemas prácticos, como por ejemplo, la clasificación de individuos o fenómenos, reconocimiento y procesamiento de imágenes, la toma de decisiones bajo incertidumbre y modelado de datos, entre otros (Liginlal y Terence, 2006; Rama y Tarres, 2007; Grabisch y Nicolas, 1994; Herrera, 2010) y las referencia en ellas mencionadas.

A continuación se presentará la definición de integral de Choquet, sus propiedades más destacadas y algunos resultados relacionados con ellas (Denneberg, 1994; Wang y Klir, 1992).

Definición 10 Sean $A \in \mathscr{A}$ y $f \in \mathscr{G}$. La integral de Choquet de $f$ con respecto a una medida difusa $\mu$ en un conjunto medible $A$, que se denota por $f_{A} f d \mu$, se define como

$$
f_{A} f d \mu=\int_{0}^{\infty} \mu\left([f]^{\alpha} \cap A\right) d \alpha .
$$

Cuando $A=X$, la integral de Choquet también puede ser indicada simplemente por $f f d \mu$.

Observe que $[f]^{\alpha},[f]^{\alpha} \cap A \in \mathscr{A}$ para todo $\alpha \in[0, \infty)$. Esto implica que $\mu\left([f]^{\alpha} \cap A\right)$ está bien definida para todo $\alpha \in[0, \infty)$. El siguiente teorema establece una forma equivalente, en términos de $[f]^{\alpha+}$, para la definición de integral de Choquet con respecto a las medidas difusas finitas (Wang y Klir, 2009).

TEOREMA 4 Sean $A \in \mathscr{A}$ y $\mu$ una medida difusa. Si $\mu(A)$ es finita, entonces para $\alpha \in[0, \infty)$ se tiene

$$
f_{A} f d \mu=\int_{0}^{\infty} \mu\left([f]^{\alpha+} \cap A\right) d \alpha .
$$

Demostración. Para algún $\varepsilon>0$, se tiene

$$
\begin{aligned}
f_{A} f d \mu & =\int_{0}^{\infty} \mu\left([f]^{\alpha} \cap A\right) d \alpha \\
& =\int_{0}^{\infty} \mu(\{x \mid f(x) \geq \alpha\} \cap A) d \alpha \\
& \geq \int_{0}^{\infty} \mu(\{x \mid f(x)>\alpha\} \cap A) d \alpha \\
& \geq \int_{0}^{\infty} \mu(\{x \mid f(x) \geq \alpha+\varepsilon\} \cap A) d \alpha \\
& =\int_{0}^{\infty} \mu(\{x \mid f(x) \geq \alpha+\varepsilon\} \cap A) d(\alpha+\varepsilon) \\
& =\int_{\varepsilon}^{\infty} \mu(\{x \mid f(x) \geq \alpha\} \cap A) d \alpha \\
& \geq \int_{0}^{\infty} \mu(\{x \mid f(x) \geq \alpha\} \cap A) d \alpha-\varepsilon \cdot \mu(A) \\
& =\int_{A} f d \mu-\varepsilon \cdot \mu(A) .
\end{aligned}
$$

Puesto $\mu(A)<\infty$ cuando $\varepsilon \rightarrow 0$, se tiene $\varepsilon$. $\mu(A) \rightarrow 0$, y en consecuencia se obtendría que

$$
\begin{aligned}
f_{A} f d \mu & =\int_{0}^{\infty} \mu(\{x \mid f(x)>\alpha\} \cap A) d \alpha \\
& =\int_{0}^{\infty} \mu\left([f]^{\alpha+} \cap A\right) d \alpha .
\end{aligned}
$$

Cuando $\mu$ es una medida difusa aditiva, la integral de Choquet coincide con la integral de Le- 
besgue. Así, la integral de Choquet puede considerarse como la generalización de la integral de Lebesgue.

EjEMPLO 9 Sean $X=[0,1], \mathscr{A}$ la $\sigma$-álgebra de Borel en $[0,1], \mu(B)=[m(B)]^{2}$ para $B \in \mathscr{A}$, donde $m$ es la medida de Lebesgue, y $f(x)=x^{2}$ para $x \in X$. En estas condiciones $\mu$ es una medida difusa en $\mathscr{A}$ y $f$ es una función medible en $X$. Aplicando la definición de la integral de Choquet de $f$ con respecto a $\mu$, se tiene que

$$
\begin{aligned}
f f d \mu & =\int_{0}^{\infty} \mu(\{x \mid f(x) \geq \alpha\}) d \alpha \\
& =\int_{0}^{\infty} \mu\left(\left\{x \mid x^{2} \geq \alpha\right\}\right) d \alpha \\
& =\int_{0}^{1} \mu([\sqrt{\alpha}, 1]) d \alpha \\
& =\int_{0}^{1}[m([\sqrt{\alpha}, 1])]^{2} d \alpha \\
& =\int_{0}^{1}(1-\sqrt{\alpha})^{2} d \alpha \\
& =\int_{0}^{1}(1-2 \sqrt{\alpha}+\alpha) d \alpha \\
& =1-\frac{1}{3}+\frac{1}{2}=\frac{1}{6} .
\end{aligned}
$$

Propiedades de la integral de Choquet: A continuación se dan algunas propiedades que satisface la integral de Choquet (Denneberg, 1994; Wang y Klir, 2009; De Campos y Bolaños, 1992; Wang y Klir, 1992).

TEOREMA 5 Sean $f$ y g funciones medibles no negativas en $(X, \mathscr{A}, \mu)$, donde $\mu$ es una medida difusa. Si $A$ y $B$ son conjuntos medibles, y a es una constante real no negativa, entonces

1. $f 1 d \mu=\mu(A)$.

2. $f f d \mu=f f \cdot \mathscr{X}_{\mathscr{A}} d \mu$.

3. Si $f \leq g$ en $A$, entonces $f f d \mu \leq f g d \mu$.

4. Si $A \subset B$, entonces $f_{A} f d \mu \leq f_{B} f d \mu$.
5. $f a \cdot f d \mu=a \cdot f f d \mu$.

6. $f(f \vee g) d \mu \geq f f d \mu \vee f g d \mu$.

7. $f(f \wedge g) d \mu \leq f f d \mu \wedge f g d \mu$

Las demostraciones de estas propiedades se obtienen aplicando la definición de integral de Choquet.

Te OREMA 6 Sean $A \in \mathscr{A}$ y $\mu$ una medida difusa. Para cualquier constante $c$ que satisfaga $f+c \geq 0$, se tiene que

$$
f_{A}(f+c) d \mu=f_{A} f d \mu+c \cdot \mu(A) \text {. }
$$

Demostración. De la definición de la integral de Choquet se tiene que $f(x)+c \geq \alpha$ para cada $x \in X$; así, cuando $\alpha$ esta entre 0 y $c$, se tiene

$$
\begin{aligned}
& f_{A}(f+c) d \mu=\int_{0}^{\infty} \mu(\{x \mid f(x)+c>\alpha\} \cap A) d \alpha \\
& =\int_{c}^{\infty} \mu(\{x \mid f(x)+c>\alpha\} \cap A) d \alpha \\
& \quad+\int_{0}^{c} \mu(\{x \mid f(x)+c>\alpha\} \cap A) d \alpha \\
& =\int_{c}^{\infty} \mu(\{x \mid f(x)>\alpha-c\} \cap A) d(\alpha-c) \\
& \quad+\int_{0}^{c} \mu(X \cap A) d \alpha \\
& =\int_{0}^{\infty} \mu(\{x \mid f(x)>\alpha\} \cap A) d \alpha \\
& \quad+\int_{0}^{c} \mu(A) d \alpha \\
& =f_{A} f d \mu+c \cdot \mu(A) .
\end{aligned}
$$

Al igual que para el caso de la integral de Sugeno, debido a la no aditividad de $\mu$ la integral de Choquet no es en general lineal con respecto a su integrando, propiedad que sí satisface la integral de Lebesgue. 
EJEMPlO $10 \operatorname{Sean} X=\{a, b\}, \mathscr{A}=\mathscr{P}(X)$ y $\mu$ una función medible sobre $X$ definida por

$$
\mu(A)= \begin{cases}0 & \text { si } A=\varnothing, \\ 1 & \text { en otros casos. }\end{cases}
$$

Considérense las funciones

$$
f(x)=\left\{\begin{array}{lll}
0 & \text { si } & x=a, \\
1 & \text { si } & x=b,
\end{array}\right.
$$

y

$$
g(x)=\left\{\begin{array}{lll}
0 & \text { si } & x=b, \\
1 & \text { si } & x=a
\end{array} .\right.
$$

Aplicando las propiedades de la integral de Choquet, se tiene que

$$
\begin{aligned}
& \begin{aligned}
f f d \mu & =\int_{0}^{\infty} \mu(\{x \mid f(x) \geq \alpha\} \cap A) d \alpha \\
& =\int_{0}^{1} \mu(\{b\}) d \mu=1 \times 1=1,
\end{aligned} \\
& \begin{aligned}
f g d \mu & =\int_{0}^{\infty} \mu(\{x \mid g(x) \geq \alpha\} \cap A) d \alpha \\
& =\int_{0}^{1} \mu(\{a\}) d \mu=1 \times 1=1
\end{aligned}
\end{aligned}
$$

y

$$
\begin{aligned}
f(f+g) d \mu & =f d \mu=\int_{0}^{\infty} \mu(\{x \mid 1 \geq \alpha\}) d \alpha \\
& =\int_{0}^{1} 1 d \mu=1 .
\end{aligned}
$$

De donde se puede concluir que

$$
f(f+g) d \mu \neq f f d \mu+f g d \mu ;
$$

esto es, en general, la integral de Choquet no satisface la linealidad.

Intentando resolver este inconveniente de la no linealidad de la integral de Choquet, Murofushi y Sugeno (1991) introducen el concepto de funciones equiordenadas. Este concepto trata de reflejar la idea de que las imágenes de las funciones $f$ y $g$ están ordenadas de la misma manera (Wang y Klir, 2009; De Campos y Bolaños, 1992; Wang y Klir, 1992; Murofushi y Sugeno, 1991).
DEFINICIÓN 11 Sean $f$ y $g$ funciones medibles no negativas. Se dice que $f$ y $g$ son equiordenadas, y se denota por $f \sim g$, si y sólo si $f\left(x_{1}\right)<f\left(x_{2}\right)$ implica que $g\left(x_{1}\right)<g\left(x_{2}\right)$ para todo $x_{1}, x_{2} \in X$.

Como consecuencia de esta definición se obtiene el siguiente resultado (Wang y Klir, 2009; De Campos y Bolaños, 1992; Wang y Klir, 1992; Murofushi y Sugeno, 1991).

TEOREMA 7 Si $f \sim g$, entonces

$$
f(f+g) d \mu=f f d \mu+f g d \mu .
$$

Más adelante se realiza una demostración de este resultado para el caso discreto (ver Proposición 10).

\section{Integrales de Sugeno y Choquet en conjuntos}

finitos: A continuación se presenta una caracterización de las integrales de Sugeno y Choquet cuando el conjunto $X$ es finito.

Sea $\mu$ una medida difusa en $X$. Sean $X=$ $\left\{x_{1}, x_{2}, x_{3}, \ldots, x_{n}\right\}$ y $f: X \rightarrow[0, \infty]$ una función con recorrido $\left\{f\left(x_{1}\right), f\left(x_{2}\right), f\left(x_{3}\right), \ldots, f\left(x_{n}\right)\right\}$ tal que $f\left(x_{1}\right) \leq f\left(x_{2}\right) \leq f\left(x_{3}\right) \leq \ldots \leq f\left(x_{n}\right)$. de

Considérese $A_{i}=\left\{x_{i}, x_{i+1}, x_{i+2}, \ldots, x_{n}\right\}$, don-

$$
\begin{aligned}
\mu\left(A_{1}\right)= & \mu\left(\left\{x_{1}, x_{2}, x_{3}, \ldots, x_{n}\right\}\right), \\
\mu\left(A_{2}\right)= & \mu\left(\left\{x_{2}, x_{3}, \ldots, x_{n}\right\}\right), \\
& \vdots \\
\mu\left(A_{n}\right)= & \mu\left(\left\{x_{n}\right\}\right) .
\end{aligned}
$$

Ahora, si $f_{i}$ indica el valor de $f$ en el punto $x_{i}$, entonces la integral de Sugeno y la integral de Choquet se pueden escribir, respectivamente, como

$$
f f d \mu=\bigvee_{i=1}^{n}\left(f_{i} \wedge \mu\left(A_{i}\right)\right)=S_{\mu}(f)
$$

y

$$
f f d \mu=\sum_{i=1}^{n} f_{i} \cdot\left(\mu\left(A_{i}\right)-\mu\left(A_{i+1}\right)\right)=\mathscr{C}_{\mu}(f),
$$

donde $A_{n+1}=\varnothing$. 
EJEMPLO 11 Sean $X=\{0,1,2\}, \mathscr{A}=\mathscr{P}(X)$ y $\mu$ la medida difusa sobre $\mathscr{A}$ definida por

$$
\mu(A)=\left\{\begin{array}{c}
|A| \text { si } A \neq\{0,1\} \\
3 \text { si } A=\{0,1\} .
\end{array}\right.
$$

Considérese ahora la función

$$
f(x)=\left\{\begin{array}{lll}
4 & \text { si } & x=0 \\
3 & \text { si } & x=1 \\
2 & \text { si } & x=2
\end{array}\right.
$$

Entonces $f_{1}=f(2)=2, f_{2}=f(1)=3$ y $f_{3}=$ $f(0)=4 ; \mu\left(A_{1}\right)=\mu(\{0,1,2\})=3, \mu\left(A_{2}\right)=$ $\mu(\{0,1\})=3$ y $\mu\left(A_{3}\right)=\mu(\{0\})=1$. Luego

$$
\begin{array}{rl}
f f & d \mu=\bigvee_{i=1}^{n}\left(f_{i} \wedge \mu\left(A_{i}\right)\right) \\
& =\left(f_{1} \wedge \mu\left(A_{1}\right)\right) \vee\left(f_{2} \wedge \mu\left(A_{2}\right)\right) \vee\left(f_{3} \wedge \mu\left(A_{3}\right)\right) \\
& =\{2 \wedge 3\} \vee\{3 \wedge 3\} \vee\{4 \wedge 1\}=1 \vee 3 \vee 2=3
\end{array}
$$

y

$$
\begin{aligned}
f f d & \mu=\sum_{i=1}^{n} f_{i} \cdot\left(\mu\left(A_{i}\right)-\mu\left(A_{i+1}\right)\right) \\
= & f_{1} \cdot\left(\mu\left(A_{1}\right)-\mu\left(A_{2}\right)\right)+f_{2} \cdot\left(\mu\left(A_{2}\right)-\mu\left(A_{3}\right)\right) \\
& +f_{3} \cdot\left(\mu\left(A_{3}\right)-\mu\left(A_{4}\right)\right) \\
= & 2 \cdot(3-3)+3 \cdot(3-1)+4 \cdot(1-0)=10 .
\end{aligned}
$$

En la Definición 11 se introdujo la noción de funciones equiordenadas; como se dijo, dicha noción trata de reflejar la idea de que las imágenes de las funciones $f$ y $g$ están ordenadas de la misma manera. A continuación se demuestra que, bajo dicha propiedad, tanto la integral de Sugeno como la integral de Choquet satisfacen una propiedad de linealidad (De Campos y Bolaños, 1992).

Proposición $9 \operatorname{Sean} f, g: X \rightarrow[0, \infty)$ funciones no negativas y $\mu$ una medida difusa en $X$. Si $f y g$ son funciones equiordenadas, entonces

$$
S_{\mu}(f \vee g)=S_{\mu}(f) \vee S_{\mu}(g)
$$

Demostración. Supongamos que

$$
f\left(x_{1}\right) \leq f\left(x_{2}\right) \leq f\left(x_{3}\right) \leq \ldots \leq f\left(x_{n}\right) .
$$

Como $f \sim g$, entonces

$$
g\left(x_{1}\right) \leq g\left(x_{2}\right) \leq g\left(x_{3}\right) \leq \ldots \leq g\left(x_{n}\right) ;
$$

también se obtiene que:

$$
\begin{aligned}
& f\left(x_{1}\right) \vee g\left(x_{1}\right) \leq f\left(x_{2}\right) \vee g\left(x_{2}\right) \\
& \quad \leq f\left(x_{3}\right) \vee g\left(x_{3}\right) \leq \ldots \leq f\left(x_{n}\right) \vee g\left(x_{n}\right) .
\end{aligned}
$$

Aplicando la definición de la integral de Sugeno, se tiene

$$
\begin{aligned}
S_{\mu}(f \vee g) & =\bigvee_{i=1}^{n}\left(\left(f_{i} \vee g_{i}\right) \wedge \mu\left(A_{i}\right)\right) \\
& =\bigvee_{i=1}^{n}\left(\left(f_{i} \wedge \mu\left(A_{i}\right)\right) \vee\left(g_{i} \wedge \mu\left(A_{i}\right)\right)\right. \\
& =\bigvee_{i=1}^{n}\left(f_{i} \wedge \mu\left(A_{i}\right)\right) \vee \bigvee_{i=1}^{n}\left(g_{i} \wedge \mu\left(A_{i}\right)\right) \\
& =S_{\mu}(f) \vee S_{\mu}(g) .
\end{aligned}
$$

Proposición 10 Sean $f, g: X \rightarrow[0, \infty)$ funciones no negativas y $\mu$ una medida difusa en $X$. Si $f y g$ son funciones equiordenadas, entonces

$$
\mathscr{C}_{\mu}(f+g)=\mathscr{C}_{\mu}(f)+\mathscr{C}_{\mu}(g) .
$$

Demostración. Supongamos que

$$
f\left(x_{1}\right) \leq f\left(x_{2}\right) \leq f\left(x_{3}\right) \leq \ldots \leq f\left(x_{n}\right) .
$$

Como $f \sim g$, entonces

$$
g\left(x_{1}\right) \leq g\left(x_{2}\right) \leq g\left(x_{3}\right) \leq \ldots \leq g\left(x_{n}\right) ;
$$

también se obtiene que:

$$
\begin{aligned}
& f\left(x_{1}\right)+g\left(x_{1}\right) \leq f\left(x_{2}\right)+g\left(x_{2}\right) \\
& \quad \leq f\left(x_{3}\right)+g\left(x_{3}\right) \leq \ldots \leq f\left(x_{n}\right)+g\left(x_{n}\right) .
\end{aligned}
$$

Ahora, sea $A_{i}=\left\{x_{i}, x_{i+1}, x_{i+2}, \ldots, x_{n}\right\}$. Aplicando la definición de la integral de Choquet se tiene

$$
\begin{aligned}
\mathscr{C}_{\mu}(f+g)= & \sum_{i=1}^{n}\left(f_{i}+g_{i}\right)\left(\mu\left(A_{i}\right)-\mu\left(A_{i+1}\right)\right) \\
= & \sum_{i=1}^{n} f_{i} \cdot\left(\mu\left(A_{i}\right)-\mu\left(A_{i+1}\right)\right) \\
& \quad+\sum_{i=1}^{n} g_{i} \cdot\left(\mu\left(A_{i}\right)-\mu\left(A_{i+1}\right)\right) \\
= & \mathscr{C}_{\mu}(f)+\mathscr{C}_{\mu}(g) .
\end{aligned}
$$


Caracterización de las integrales de Sugeno y Choquet: A continuación se presentan dos resultados que permiten caracterizar las integrales de Sugeno y Choquet por medio del cumplimiento de ciertas propiedades (De Campos y Bolaños, 1992). Se inicia con un resultado relacionado con la integral de Sugeno.

TEOREMA 8 Un funcional E no negativo, definido para funciones con valores en $[0,1]$, satisface las siguientes condiciones:

(a) si $f \sim g$, entonces $E(f \vee g)=E(f) \vee E(g)$ ( $F$-aditividad de orden);

(b) sif $\leq g$, entonces $E(f)=E(g)$ (monotonía);

(c) $E\left(I_{x}\right)=1$ (normalización);

(d) $\forall a \in(0,1], E(a \wedge f)=a \wedge E(f)$ (homogeneidad),

si y sólo si existe una sola medida difusa normalizada $\mu$ tal que E es la integral de Sugeno con respecto $a \mu$.

Demostración. La suficiencia se sigue de las propiedades de la integral de Sugeno y por la Proposición 9.

Necesidad: Sea la función definida por $\mu(A)=$ $E\left(I_{A}\right), \forall A \subseteq X$ se mostrará que $\mu$ es una medida difusa normalizada.

La monotonía se deduce de la propiedad $(b)$; $\mu(X)=1$ se tiene de la propiedad (c).

Para todo $a \in(0,1]$,

$$
a=E(a)=E\left(a \wedge I_{x}\right)=a \wedge E\left(I_{x}\right)=a \wedge 1=a,
$$

por las condiciones $(c)$ y $(d)$; entonces, por $(a)$ se tiene que para todo $a \in(0,1]$,

$$
a=E(a)=E\left(a \wedge 0_{x}\right)=E(a) \wedge E\left(0_{X}\right)=a \wedge E\left(O_{X}\right) \text {. }
$$

Por lo tanto, $E\left(O_{X}\right) \leq a, \forall a \in(0,1]$, y se obtiene que $\mu(\varnothing)=E\left(0_{x}\right)=0$, porque $E$ es un funcional no negativo.

Se mostrará ahora que $E(\cdot)$ coincide con $S_{\mu}(\cdot)$.

Sea $f: X \rightarrow[0,1]$ y supóngase que

$$
f\left(x_{1}\right) \leq f\left(x_{2}\right) \leq f\left(x_{3}\right) \leq \ldots \leq f\left(x_{n}\right) .
$$

Entonces $f$ se puede escribir como:

$$
f(x)=\bigvee_{i=1}^{n} t_{i}(x),
$$

donde $t_{i}(x)=f_{i} \wedge I_{A_{i}}$,

$$
A_{i}=\left\{x_{i}, x_{i+1}, x_{i+2}, \ldots, x_{n}\right\}, i=1, \ldots, n .
$$

Se puede verificar que $t_{j} \sim t_{i} \forall i, j=1,2,3 \ldots, n$. Ahora,

$$
\begin{aligned}
E(b) & =E\left(\bigvee_{i=1}^{n}\right) E\left(t_{i}\right)=\bigvee_{i=1}^{n} E\left(f_{i} \wedge I_{A_{i}}\right) \\
& =\bigvee_{i=1}^{n}\left(f_{i} \wedge E\left(I_{A_{i}}\right)\right)=\bigvee_{i=1}^{n}\left(f_{i} \wedge \mu\left(A_{i}\right)\right) .
\end{aligned}
$$

Entonces $E$ coincide con la integral de Sugeno con respecto a $\mu$.

En conclusión: la integral de Sugeno se caracteriza por ser un funcional $F$-aditivo, monótono y homogéneo.

Ahora, se mostrará una caracterización análoga para la integral se Choquet.

TEOREMA 9 Un funcional E definido sobre funciones no negativas satisface las siguientes condiciones:

(a) si $f \sim g$, entonces $E(f+g)=E(f)+E(g)$ (aditividad de orden);

(b) sif $\leq g$, entonces $E(f) \leq E(g)$ (monotonía);

(c) $E\left(I_{x}\right)=1 \quad$ (normalización);

(d) $\forall a \geq 0, E(a \cdot f)=a \cdot E(f)$ (homogeneidad),

si y sólo si existe una sola medida difusa normalizada $\mu$ tal que E es la integral de Choquet con respecto a la medida $\mu$.

Demostración. Necesidad: Se define la función $\mu(A)=E\left(I_{A}\right), \forall A \subseteq X$. Se probará que $\mu$ es una medida difusa normalizada. 
1. $\mu(X)=E\left(I_{x}\right)=1$ se tiene por la condición $(c)$.

2. $\mu(\varnothing)=E\left(I_{\varnothing}\right)=E\left(O_{x}\right)=0$, se tiene por la siguiente razón:

Como $O_{x}$ es una función constante $O_{x}=f$ para cada función $f$, por la condición $(a)$, se tiene

$$
E(f)=E\left(f+0_{x}\right)=E(f)+E\left(0_{x}\right) ;
$$

entonces $E\left(O_{x}\right)=0$.

3. Si $A \subseteq B$, entonces $I_{A}(x) \leq I_{B}(x), \forall x \in X$, se tiene por $(b) ; \mu(A)=E\left(I_{A}\right) \leq E\left(I_{B}\right)=$ $\mu(B)$. Luego $\mu$ es una medida difusa normalizada.

Ahora se probará que el funcional es la integral de Choquet.

Sea $f$ una función no negativa y que verifica

$$
f\left(x_{1}\right) \leq f\left(x_{2}\right) \leq f\left(x_{3}\right) \leq \ldots \leq f\left(x_{n}\right)
$$

entonces $f$ se puede expresar como

$$
f(x)=\sum_{i=1}^{n} \phi_{i}(x)
$$

donde

$$
\begin{gathered}
\phi_{i}(x)=\left(f_{i}-f_{i-1}\right) I_{A}(x), i=2, \ldots, n, \\
\operatorname{con} \phi_{1}(x)=f_{1} I_{A_{1}}(x) \mathrm{y} \\
A_{i}=\left\{x_{i}, x_{i+1}, x_{i+2}, \ldots, x_{n}\right\}, i=2, \ldots, n .
\end{gathered}
$$

Cada par de funciones $\phi_{i}$ son equiordenadas; utilizando $(a)$ y $(d)$ se tiene

$$
\begin{aligned}
E(f) & =E\left(\sum_{i=1}^{n} \phi_{i}\right)=\sum_{i=1}^{n} E\left(\phi_{i}(x)\right) \\
& =E\left(f_{1} I_{A_{1}}\right)+\sum_{i=2}^{n} E\left(\left(f_{i}-f_{i-1}\right)\right) I_{A} \\
& =f_{1} E\left(I_{A_{1}}\right)+\sum_{i=2}^{n}\left(\left(f_{i}-f_{i-1}\right)\right) E\left(I_{A}\right) \\
& =f_{1}+\sum_{i=2}^{n}\left(\left(f_{i}-f_{i-1}\right)\right) \mu\left(A_{i}\right),
\end{aligned}
$$

$$
\begin{aligned}
& =f_{n} \mu\left(A_{n}\right)+\sum_{i=2}^{n-1} f_{i} \cdot\left(\mu\left(A_{i}\right)-\mu\left(A_{i+1}\right)\right) \\
& =\mathscr{C}_{\mu}(f) .
\end{aligned}
$$

Suficiencia: Las condiciones $(b),(c)$ y $(d)$ son propiedades conocidas de $\mathscr{C}_{\mu}(\cdot)$, y la condición $(a)$ fue probada en la Proposición 10.

En conclusión: la integral de Choquet se caracteriza por ser un funcional equiordenado, monótono y homogéneo.

Como se ve, las propiedades son completamente paralelas entre las integrales de Choquet y Sugeno: ambas integrales se ajustan al mismo modelo formal, pero difieren en la utilización de operadores (suma y producto para integral de Choquet, máximo y mínimo para la integral de Sugeno). Sin embargo, las propiedades matemáticas de estos operadores dan a cada funcional características particulares que los hacen útiles en diferentes contextos.

Existen otras analogías entre $\mathscr{C}_{\mu}(\cdot)$ y $S_{\mu}(\cdot)$. Por ejemplo, la medida de posibilidad con la integral de Sugeno se comporta de manera similar a la integral de Choquet para el caso de las medidas de probabilidad.

Si la medida difusa es de probabilidad $(P)$, entonces la integral de Choquet coincide con la esperanza matemática con respecto a $P$, y por lo tanto se puede escribir como:

$$
\mathscr{C}_{p}(f)=\sum_{i=1}^{n} p_{i} \cdot f_{i}, \quad i=1,2,3, \ldots, n .
$$

La siguiente proposición da una expresión análoga a la anterior para $S_{\mu}(\cdot)$ cuando $\mu$ es una medida de posibilidad.

PROPOSICIÓN 11 Sea $\Pi$ una medida posibilidad y $f: X \rightarrow[0,1]$ una función. La integral de Sugeno de $f$ con respecto a $\Pi$ puede escribirse como:

$$
S_{\Pi}(f)=\bigvee_{i=1}^{n}\left(\Pi_{i} \wedge f_{i}\right),
$$

donde $\Pi_{i}=\Pi\left(x_{i}\right), i=1,2,3, \ldots, n$.

Nótese el paralelismo que existe entre la probabilidad y la posibilidad con respecto a las integrales de Choquet y Sugeno, respectivamente. 


\section{Algunas aplicaciones de las integrales y las me- didas difusas}

La teoría clásica de la medida, basada en medidas aditivas y asociadas a la integral de Lebesgue, ha sido muy importante, no solo en el análisis matemático, sino también ha desempeñado un papel importante en diferentes campos de aplicación. Tal vez el papel más importante han sido la teoría de probabilidad y las ecuaciones diferenciales parciales.

Como ya se mencionó, se ha reconocido que la teoría clásica, a pesar de sus muchas aplicaciones, está limitada por el requisito de la aditividad de las medidas. Es por ello que han surgido las medidas difusas y, ligadas a ellas, las integrales difusas. En los últimos años, la teoría de medidas difusas e integrales difusas se han convertido en una rama de la matemática que ha captado un gran interés de investigación.

Veamos a continuación algunos ejemplos donde se aplican las medidas difusas y las integrales difusas (Ramírez-Lamus, 2012).

EJEMPLO 12 Considere el problema de la evaluación de calidad de un plato $P$ de cocina. Supóngase que los factores de calidad que se consideran son: el sabor, el olor y la apariencia (incluyendo, por ejemplo, el color, la forma, etc.). Se denotan estos factores por $S, O$ y $A$, entonces $X=\{S, O, A\}$. Se emplea como medida de importancia: $\mu(\{S\})=0,7, \mu(\{O\})=0,1, \mu(\{A\})=0$, $\mu(\{S, O\})=0,9, \mu(\{A, S\})=0,8, \mu(\{O, A\})=0,3$, $\mu(X)=1$ y $\mu(\varnothing)=0$.

Es claro que $\mu$ no es aditiva. Se invita a expertos como árbitro para juzgar el factor de calidad de un determinado plato, donde los factores de calidad son dados de la siguiente manera: $f(S)=0,9$, $f(O)=0,6$ y $f(A)=0,8$. Entonces la evaluación de la calidad del plato $P$ se calcula como sigue:

$$
\begin{aligned}
P= & S_{\mu}(f) \\
= & \left\{0,6 \wedge \mu\left([f]^{0,6}\right)\right\} \vee\left\{0,8 \wedge \mu\left([f]^{0,8}\right)\right\} \\
& \vee\left\{0,9 \wedge \mu\left([f]^{0,9}\right)\right\} \\
= & \{0,6 \wedge \mu(X)\} \vee\{0,8 \wedge \mu(\{S, A\}) \\
& \vee\{0,9 \wedge \mu(\{S\})\} \\
= & \{0,6 \wedge 1\} \vee\{0,8 \wedge 0,8\} \vee\{0,9 \wedge 0,7\} \\
= & 0,6 \vee 0,8 \vee 0,7=0,8 .
\end{aligned}
$$

Entonces, con los criterios dados, la evaluación de calidad del plato de cocina $P$ es del $80 \%$.

El siguiente es un ejemplo donde se utilizan las $\lambda$-medidas y la integral de Choquet para realizar la selección de individuos que aspiran ingresar a una universidad (Ramírez-Lamus, 2012).

EJEMPLO 13 Una Universidad en su proceso de selección de estudiantes utiliza las Pruebas de Estado como criterio de clasificación. Si un estudiante desea ingresar a una carrera de Ingeniería, la universidad le da un porcentaje a las siguientes materias:

Matemáticas: $45 \%$, Lenguaje: $45 \%$ y Física: $30 \%$ Denótese: Matemáticas con $x_{1}$, Lenguaje con $x_{2}$, Física con $x_{3}$ y cuatro estudiantes con $c_{1}, c_{2}, c_{3} \mathrm{y}$ $c_{4}$ respectivamente. Los puntos de cada materia están entre 0-100. Según el anterior criterio ¡cuál será la clasificación de los estudiantes si tienen los siguientes puntajes?

Estudiante $c_{1}: x_{1}=45, x_{2}=50$ y $x_{3}=40$.

Estudiante $c_{2}: x_{1}=56, x_{2}=35$ y $x_{3}=50$.

Estudiante $c_{3}: x_{1}=39, x_{2}=58$ y $x_{3}=55$.

Estudiante $c_{4}: x_{1}=58, x_{2}=38$ y $x_{3}=57$.

Entonces $X=\left\{x_{1}, x_{2}, x_{3}\right\}$. El grado de importancia es dado por $g_{1}=g_{\lambda}\left(\left\{x_{1}\right\}\right)=0,45$, $g_{2}=g_{\lambda}\left(\left\{x_{2}\right\}\right)=0,45$ y $g_{3}=g_{\lambda}\left(\left\{x_{3}\right\}\right)=0,3$.

Se debe encontrar la $\lambda$-medida apropiada para este problema. Para ello se considera que $g_{\lambda}(X)=$ 1 y se utiliza la ecuación (3), de donde se obtiene que

$$
\begin{aligned}
\lambda+1 & =\prod_{i=1}^{n}\left(\lambda g_{i}+1\right) \\
& =(0,45 \lambda+1)(0,45 \lambda+1)(0,3 \lambda+1) .
\end{aligned}
$$

Esto implica que $0,06067 \lambda^{3}+0,4725 \lambda^{2}+0,2 \lambda=0$. Las raíces aproximadas de esta ecuación son: 0 , $-7,3388$ y $-0,44919$.

Cuando $\lambda=0$ la medida es aditiva; en este caso la clasificación se realizaría por medio del promedio ponderado de cada aspirante.

Lo interesante es considerar el caso en el cual la medida no sea aditiva; eso se obtiene cuando $\lambda=-0,44919$ (el valor $-7,3388$ no se considera, dado que no pertenece al intervalo $(-1, \infty))$. En este caso se necesita saber la medida de cada elemento de la $\sigma$-álgebra $\mathscr{P}(X)$, para lo cual se utiliza 
la ecuación (2), de donde se obtiene:

$$
\begin{aligned}
g_{\lambda}\left(\left\{x_{1}, x_{2}\right\}\right)= & g_{\lambda}\left(\left\{x_{1}\right\}\right)+g_{\lambda}\left(\left\{x_{2}\right\}\right) \\
& +\lambda \cdot g_{\lambda}\left(\left\{x_{1}\right\}\right) \cdot g_{\lambda}\left(\left\{x_{2}\right\}\right)=0,8090, \\
g_{\lambda}\left(\left\{x_{1}, x_{3}\right\}\right)= & g_{\lambda}\left(\left\{x_{1}\right\}\right)+g_{\lambda}\left(\left\{x_{3}\right\}\right) \\
& +\lambda \cdot g_{\lambda}\left(\left\{x_{1}\right\}\right) \cdot g_{\lambda}\left(\left\{x_{3}\right\}\right)=0,6894, \\
g_{\lambda}\left(\left\{x_{2}, x_{3}\right\}\right)= & g_{\lambda}\left(\left\{x_{2}\right\}\right)+g_{\lambda}\left(\left\{x_{3}\right\}\right) \\
& +\lambda \cdot g_{\lambda}\left(\left\{x_{2}\right\}\right) \cdot g_{\lambda}\left(\left\{x_{3}\right\}\right)=0,6894, \\
g_{\lambda}(X)= & 1 .
\end{aligned}
$$

Conociendo la medida de los elementos de la $\sigma$ álgebra, y que $f$ es el puntaje en cada asignatura para cada estudiante, se puede encontrar la integral de Choquet para cada estudiante $c_{i}$ como sigue:

$$
c_{i}=\mathscr{C}_{g_{\lambda}}(f)=\sum_{i=1}^{n}\left(f\left(x_{i}\right)-f\left(x_{i-1}\right)\right) g_{\lambda}\left(A_{i}\right) .
$$

Por lo tanto, se tiene

$$
\begin{aligned}
& c_{1}= \mathscr{C}_{g_{\lambda}}(f)=f\left(x_{3}\right) \cdot g_{\lambda}\left(\left\{x_{1}, x_{2}, x_{3}\right\}\right) \\
&+\left(f\left(x_{1}\right)-f\left(x_{3}\right)\right) \cdot g_{\lambda}\left(\left\{x_{1}, x_{2}\right\}\right) \\
& \quad+\left(f\left(x_{2}\right)-f\left(x_{1}\right)\right) \cdot g_{\lambda}\left(\left\{x_{2}\right\}\right)=46,295 . \\
& \\
& c_{2}= \mathscr{C}_{g_{\lambda}}(f)=f\left(x_{2}\right) \cdot g_{\lambda}\left(\left\{x_{1}, x_{2}, x_{3}\right\}\right) \\
&+\left(f\left(x_{3}\right)-f\left(x_{2}\right)\right) \cdot g_{\lambda}\left(\left\{x_{1}, x_{2}\right\}\right) \\
& \quad+\left(f\left(x_{1}\right)-f\left(x_{2}\right)\right) \cdot g_{\lambda}\left(\left\{x_{1}\right\}\right)=48,041 . \\
& \\
& c_{3}=\mathscr{C}_{g_{\lambda}}(f)=f\left(x_{1}\right) \cdot g_{\lambda}\left(\left\{x_{1}, x_{2}, x_{3}\right\}\right) \\
&+\left(f\left(x_{3}\right)-f\left(x_{1}\right)\right) \cdot g_{\lambda}\left(\left\{x_{2}, x_{3}\right\}\right) \\
& \quad+\left(f\left(x_{2}\right)-f\left(x_{3}\right)\right) \cdot g_{\lambda}\left(\left\{x_{2}\right\}\right)=51,3804 . \\
& \quad \\
& c_{4}=\mathscr{C}_{g_{\lambda}}(f)=f\left(x_{2}\right) \cdot g_{\lambda}\left(\left\{x_{1}, x_{2}, x_{3}\right\}\right) \\
&+\left(f\left(x_{3}\right)-f\left(x_{2}\right)\right) \cdot g_{\lambda}\left(\left\{x_{3}, x_{2}\right\}\right) \\
& \quad+\left(f\left(x_{1}\right)-f\left(x_{2}\right)\right) \cdot g_{\lambda}\left(\left\{x_{1}\right\}\right)=51,5481 .
\end{aligned}
$$

A partir de los resultados obtenidos para cada $c_{i}$, se puede concluir que la clasificación de los estudiantes es $c_{4}>c_{3}>c_{2}>c_{1}$. Es decir, que el mejor clasificado para ingresar a la carrera de Ingeniería es el estudiante $c_{4}$.
Como se puede observar en los ejemplos realizados, la aplicación de las integrales difusas y las medidas difusas van de la mano. Recientemente Liginlal y Terence (2006) realizan un estudio de las aplicaciones de las medidas e integrales difusas en las ingenierías y las ciencias sociales, dicho estudio ayuda a discernir cinco áreas:

1. La evaluación subjetiva, la previsión y la toma de decisiones.

2. La recuperación de la información.

3. El modelado de datos.

4. El análisis de actitudes y patrones.

5. Reconocimiento y clasificación.

A continuación se describen algunas situaciones donde se aplican las medidas difusas y las integrales difusas (MF Anderson et al., 2010; DT Anderson et al., 2011; Liginlal y Terence, 2006; Rama y Tarres, 2007; Grabisch y Nicolas, 1994; Herrera, 2010; Wang et al., 2000).

Método para la detección de caras basado en integrales difusas: Un método detector de caras compuesto de un conjunto de clasificadores basados en integrales difusas es presentado por Rama y Tarres (2007). Este método presenta una mejora significativa respecto del detector de caras utilizado con otras técnicas. El clasificador basado en integrales difusas mapea el conjunto de datos de entrada en un único escalar, luego, dependiendo de un cierto umbral, el valor clasificará la cara.

Sistemas de visión estereoscópica en entornos forestales: En las últimas décadas se han venido utilizando de forma manual los sistemas de visión estereoscópica para captar información del entorno en diferentes aplicaciones. Las imágenes son obtenidas mediante un sistema óptico basado en los denominados lentes de ojo de pez. Su interés se centra en obtener información de los troncos de los árboles a partir de imágenes estereoscópicas, y con las medidas obtenidas se realiza el estudio sobre el volumen de la madera, la densidad de los árboles y su evolución o crecimiento, entre otros 
(Herrera, 2010). En este sistema utilizan las integrales de Sugeno y las integrales de Choquet en el procesamiento de imágenes.

Teoría de la evidencia: Esta teoría fue desarrollada inicialmente por Dempster y después extendida por Shafer. Se considera como una extensión de la medida de probabilidad para describir la incertidumbre asociada a una evidencia.

Como ya se mencionó, la teoría de la evidencia se centra en la credibilidad que se le asigna a que un evento pueda ocurrir desde el punto de vista de la experiencia de la persona que toma las decisiones, a diferencia de la medida de probabilidad, que supone la existencia de valores asociados a los eventos determinados, los cuales son independientemente del observador. La teoría de DempsterShafer se centra en dos medidas difusas, la plausibildad y la credibilidad (Shafer, 1976; Dempster, 1967a, 1967b).

Estimación de la edad de la muerte de un individuo: La estimación de la muerte de un individuo es importante para las ciencias forense y para los antropólogos. Los métodos actuales de reconocimiento son poco confiables, debido a la variación del esqueleto y los factores tafonómicos. Los métodos multifactoriales son mejores que los métodos individuales cuando se determina edad de muerte de un individuo. Sin embargo, los métodos multifactoriales son difíciles de aplicar en esqueletos mal conservados, y rara vez proporcionan información fiable al investigador sobre su estudio. La integral de Sugeno se utiliza como método multifactorial para el estimar la edad de muerte del esqueleto de un individuo. La integral de Sugeno es más eficiente, dado que no necesita el uso de una población y gráficamente es fácil de interpretar (MF Anderson et al., 2010; DT Anderson et al., 2011).

\section{Conclusión}

El concepto de medida difusa busca generalizar el concepto de medida, al sustituir la propiedad de aditividad de las medidas clásicas por propiedades menos rígidas. Ligado a este concepto se encuentra el de integrales difusas, las cuales se definen como las integrales con respecto a una medida difusa. Dos de las integrales difusas más destacadas son la de Sugeno y la de Choquet. Dado que ofrecen un enfoque más flexible y realista a una amplia variedad de problemas, las medidas difusas, así como las integrales difusas, vienen siendo aplicadas en diferentes disciplinas, entre las que se destacan la economía, la informática, la teoría de juegos, el procesamiento de imágenes y los procesos de clasificación y evaluación de la calidad.

\section{Agradecimientos}

Queremos extender nuestro agradecimiento a la Escuela de Matemáticas de la Universidad Industrial de Santander por el soporte académico y de infraestructura dado en la realización de este trabajo. De igual forma agradecemos a los árbitros, ya que con los valiosos comentarios y sugerencias, enriquecieron y mejoraron este trabajo. Los autores declaramos que no existen conflictos de interés en la realización de este trabajo.

\section{Referencias}

Anderson DT, Keller J, Anderson MF, Wescott DJ (2011) Linguistic description of adult skeletal ageat-death estimations from fuzzy integral acquired fuzzy sets. IEEE International Conference on Fuzzy Systems 1:2274-2281

Anderson MF, Anderson DT, Wescott DJ (2010) Estimation of adult skeletal age-at-death using the Sugeno fuzzy integral. American Journal of Physical Anthropology 142(1):30-41

Asahina S, Uchino K, Murofushi T (2006) Relationship among continuity conditions and null-additivity conditions in non-additive measure theory. Fuzzy Sets and Systems 157:691-698

Ash RB (2000) Probability and measure theory. Academy Press, London

Ayyub BM, Klir GJ (2006) Uncertainty modeling and analysis in engineering and the sciences. Kluwer Academic Publishers, Massachusetts, USA

Banon G (1981) Distinction between several subsets of fuzzy measures. Fuzzy Sets and Systems 5:291-305

De Campos LM, Bolaños MJ (1992) Characterization and comparison Sugeno and Choquet integrals. Information and Control 52:61-67 
Dempster AP (1967a) Upper and lower probabilities induced by multi-valued mapping. Annals of Mathematical Statistics 38:325-339

Dempster AP (1967b) Upper and lower probability inferences based on a sample from a finite univariate population. Biometrika 54:515-528

Denneberg D (1994) Non-additive measure and integral. Kluwer Academic Publishers, Dordrecht

Folland GB (1999) Real analysis: modern techniques and their applications (2nd ed.). John Wiles \& Sons, New York

Garmendia L (2001) Contribución al estudio de las medidas en la lógica borrosa: condicionalidad, especifícidad y transitividad. Tesis Doctoral, Universidad Politécnica de Madrid, Madrid, España

Garmendia L (2005) The evolution of the concept of fuzzy measure. Intelligent Data Mining. Studies in Computational Intelligence 5:185-200

Geronimo JR (1988) Medidas fuzzy. Tesis de Maestría, Universidade Estadual de Campinas, Campinas, Brazil

Grabisch M, Nicolas J (1994) Classification by fuzzy integral: performance and tests. Fuzzy Sets and Systems 65:225-271

Herrera PJ (2010) Correspondencia estereoscópica en imágenes obtenidas con proyección omnidireccional para entornos forestales. Tesis Doctoral, Universidad Complutense de Madrid, Madrid, España

Liginlal D, Terence TO (2006) Modeling attitude to risk in human decision processes: An application of fuzzy measures. Fuzzy Sets and Systems 157:30403054

Murofushi T (1987) Two approaches to fuzzy measure theory: integrals based on pseudo-addition a Choquet's integral. Tesis Doctoral, Tokyo Institute of Technology, Yokohama, Japan

Murofushi T, Sugeno M (1991) A theory of fuzzy measures: representations, the Choquet integral and null sets. Journal of Mathematical Analysis and Applications 159:532-549

Murofushi T, Sugeno M (2000) Fuzzy measures and fuzzy integrals. Department of Computational Intelligence and Systems Science, Tokyo Institute of Technology, Yokohama, Japan

Nguyen HT, Walter EA (2006) A first course in fuzzy logic (Third ed.). Chapman \& Hall

Rama A, Tarres F (2007) Un nuevo método para la detección de caras basado en integrales difusas.
XXII Simposium Nacional de la Unión Científica Internacional de Radio

Ramírez-Lamus ER (2012) Medidas difusas. Tesis de Maestría, Universidad Industrial de Santander, Bucaramanga, Colombia

Román-Flores H, Flores-Franulic A, Chalco-Cano Y (2007) The fuzzy integral for monotone functions. Applied Mathematics and Computation 185(1):492498

Shafer G (1976) A mathematical theory of evidence. Princeton University Press, Princeton, New Jersey

Song J (2005) On generalization of converge theorems in non-additive measure theory. Tesis Doctoral, Chiba University, Chiba, Japan

Song J, Li J (2005) Lebesgue theorems in non-additive measure theory. Fuzzy Sets and Systems 149:543-548

Sugeno M (1974) Theory of fuzzy integrals and its applications. Tesis Doctoral, Tokyo Institute of Technology, Tokyo, Japan

Sun Q (1992) On the pseudo-autocontinuity of fuzzy measures. Fuzzy Sets and Systems 45:59-68

Trillas E, Alsina C (1999) A reflection on what is a membership function. Mathware Soft Computing IV(2-3):201-215

Wang Z (1984) The autocontinuity of set function and fuzzy integral. Journal of Mathematical Analysis and Applications 99:195-218

Wang Z (1985) Asymptotic structural characteristics of fuzzy measure and their applications. Fuzzy Sets and Systems 16(3):277-290

Wang Z (1992) On the null-additivity and the autocontinuity of fuzzy measure. Fuzzy Sets and Systems 45:223-226

Wang Z, Klir G (1992) Fuzzy measure theory. Plenum Press, New York

Wang Z, Klir G (2009) Generalized measure theory. Springer, New York

Wang Z, Leung KS, Wang J (2000) Determining nonnegative monotone set functions based on Sugeno's integral: an application of genetic algorithms. Fuzzy Sets and Systems 112:155-164

Weber S (1984) $\perp$-descomposable measures and integrals for archimedean $t$-conorms $\perp$. Journal of Mathematical Analysis and Applications 101:114-138

Zadeh LA (1965) Fuzzy sets. Information and Control 8:338-353

Zadeh LA (1978) Fuzzy sets as basis for a theory of possibility. Information and Control 1:338-353 